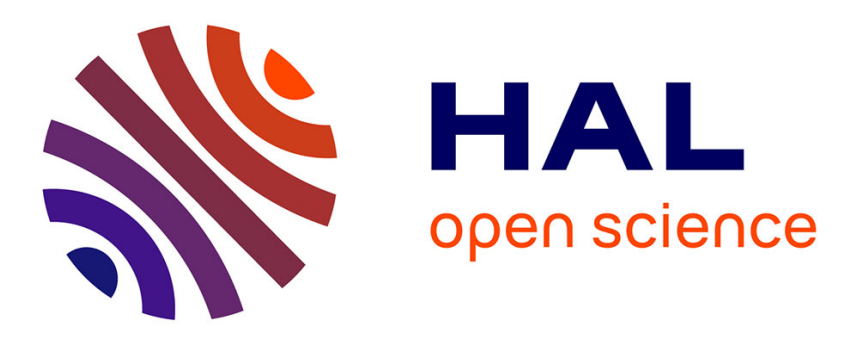

\title{
Nearwell local space and time refinement in reservoir simulation
}

\author{
Walid Kheriji, Roland Masson, Arthur Moncorgé
}

\section{To cite this version:}

Walid Kheriji, Roland Masson, Arthur Moncorgé. Nearwell local space and time refinement in reservoir simulation. Mathematics and Computers in Simulation, 2014, Volume 118, pp. 273-292. 10.1016/j.matcom.2014.11.022 . hal-00974264

\section{HAL Id: hal-00974264 \\ https://hal.science/hal-00974264}

Submitted on 7 Apr 2014

HAL is a multi-disciplinary open access archive for the deposit and dissemination of scientific research documents, whether they are published or not. The documents may come from teaching and research institutions in France or abroad, or from public or private research centers.
L'archive ouverte pluridisciplinaire HAL, est destinée au dépôt et à la diffusion de documents scientifiques de niveau recherche, publiés ou non, émanant des établissements d'enseignement et de recherche français ou étrangers, des laboratoires publics ou privés. 


\title{
NEARWELL LOCAL SPACE AND TIME REFINEMENT IN RESERVOIR SIMULATION
}

\author{
W. Kheriji ${ }^{1}$, R. MAsson ${ }^{2}$ And A. Moncorgé ${ }^{3}$
}

\begin{abstract}
In reservoir simulations, nearwell regions usually require finer space and time scales compared with the remaining of the reservoir domain. We present a domain decomposition algorithm for a two phase Darcy flow model coupling nearwell regions locally refined in space and time with a coarser reservoir discretization. The algorithm is based on an optimized Schwarz method using a full overlap at the coarse level. The main advantage of this approach is to apply to fully implicit discretizations of general multiphase flow models and to allow a simple optimization of the interface conditions based on a single phase flow equation.
\end{abstract}

2010 AMS Subject Classification. 76S05,76T10,65M55,65N55.

Mars 2014.

\section{INTRODUCTION}

Nearwell regions in reservoir simulations usually require fine space and time scales due to several physical processes such as higher Darcy velocities, the coupling of the stationary well model with the transient reservoir model, high non linearities due to phase appearance (typically gas), complex physics such as formation damage models. In addition the nearwell geological model is usually finer in the nearwell region due to available data.

If Local Grid Refinement (LGR) is commonly used in reservoir simulations in the nearwell regions, current commercial simulators still make use of a single time stepping on the whole reservoir domain. It results that the time step is globally constrained both by the nearwell small refined cells and by the high Darcy velocities and high non linearities in the nearwell region. A Local Time Stepping (LTS) with a small time step in the nearwell regions and a larger time step in the reservoir region is clearly a promising field of investigation in order to save CPU time. It is a difficult topic in the context of reservoir simulation due to the implicit time integration, and to the coupling between a mainly elliptic or parabolic unknown, the pressure, and mainly hyperbolic unknowns, the saturations and compositions.

Different approaches combining LTS and LGR have been studied for reservoir simulation applications. The first class of algorithms belongs to Domain Decomposition Methods (DDM). Matching conditions are defined at the nearwell reservoir interface with possible overlap, and a Schwarz algorithm is used to compute the solution. In the context of LTS, such DDM algorithms were first analysed by Ewing and Lazarov [5] for parabolic problems in terms of stability, error estimates and convergence of the domain decomposition method. Then, Mlacnik and Heinemann $[9,10]$ have proposed an extension to multiphase Darcy flow reservoir models using a sequential (non iterative) approach. They first compute, on the global LGR grid and with the coarse time step, an approximate solution using a simplified model. Then, the solution is computed on the nearwell region and with the fine time step using Dirichlet boundary conditions given by the previous step at the reservoir nearwell region interface. This approach is improved in [7] using a predictor corrector strategy. The predictor is the Mlacnik step, and it is followed by Dirichlet Neumann iterations until a convergence criteria on the matching conditions is achieved.

The second class of methods use both the coarse grid on the full domain and the LGR nearwell grid. These grids communicate both at the reservoir nearwell interface and also between the perforated fine and coarse cells (well interface). In the Eclipse simulator [1] a sequential version (usually called windowing) of this approach is implemented with Neumann or Dirichlet boundary conditions at the nearwell reservoir interface and at the well interface.

We propose to combine this latter windowing approach with a DDM Robin-Neumann algorithm coupling the coarse grid solution with full overlap to the refined (in space and time) nearwell grid solution. An efficient

Keywords and phrases: Darcy flow, Porous media, two phase Darcy flow, nearwell refinement, local time stepping, domain decomposition, optimized Schwarz methods.

${ }^{1}$ University Nice Sophia Antipolis 06108 Nice Cedex 02, and team Coffee INRIA Sophia Antipolis Méditerranée (Walid.Kheriji@unice.fr)

2 University Nice Sophia Antipolis 06108 Nice Cedex 02, and team Coffee INRIA Sophia Antipolis Méditerranée(Roland.Masson@unice.fr)

3 TOTAL CSTJF - 64018 Pau Cedex(arthur.moncorge@total.com) 
iterative algorithm is obtained using at the nearwell reservoir interface optimized Robin conditions for the pressure and Dirichlet conditions for the saturations and compositions. At the well interface, a Neumann condition is imposed for the pressure (assuming to fix ideas that the well condition is a fixed pressure) and input Dirichlet conditions are imposed for saturations and compositions.

The optimization of the Robin coefficients is done on a single phase flow parabolic equation for the reference pressure using existing theory for optimized Schwarz methods (see [8]), while the algorithm is applied on fully implicit discretization of two phase Darcy flows.

This paper is organized as follows. Section 2 describes the construction of our Domain Decomposition algorithm which is presented to fix ideas in the case of a gas injection through a multi-perforated well in a closed reservoir saturated with water. Section 3 assesses the efficiency of our DDM algorithm on two 3D test cases both in terms of accuracy and CPU time compared with the reference solution obtained using the LGR grid with the global fine time stepping. Our Robin-Neumann DDM algorithm is also compared with the classical windowing algorithm [1]. The first test case simulates the injection of gas through a multi-perforated well in a close reservoir saturated with water taking into account the gas dissolution in the liquid phase. The second test case models the production of a gas condensate through a multi-perforated vertical well taking into account the appearance of the oil phase in the nearwell region.

\section{Domain Decomposition Method (DDM) for a two phase Darcy flow model}

\subsection{Simplified two phase flow model}

To simplify the presentation of the DDM algorithms, we will consider in the following the example of an immiscible compressible two phase Darcy flow not taking into account the capillary pressure. The algorithms presented in this section readily extend to more complex models such as multi-phase compositional models with capillary pressure. To fix ideas the model describe the injection of a phase 2 (gas) in a $3 \mathrm{D}$ reservoir $\Omega_{r}$ initially saturated with a phase 1 (liquid) through a vertical well. Let us denote by $\Gamma_{r}$ the outer boundary of the reservoir (cf figure 1) assumed to be impervious, and by $\Gamma_{w}$ the inner boundary of the reservoir corresponding to the boundary of the vertical well. The velocities of the phases are given by the two phase Darcy laws

$$
\mathbf{V}^{(\alpha)}=-\frac{k_{r}^{(\alpha)}\left(s^{(\alpha)}\right)}{\mu^{(\alpha)}} \mathbf{K}\left(\nabla p-\rho^{(\alpha)}(p) \mathbf{g}\right), \alpha=1,2,
$$

where $\mathbf{g}$ is the gravity vector, $p$ is the pressure, $s^{(\alpha)}, \alpha=1,2$ are the phase volume fractions called saturations, $k_{r}^{(\alpha)}\left(s^{(\alpha)}\right)$ are the relative permeabilities, and $\mu^{(\alpha)}, \alpha=1,2$ are the phase viscosities assumed to be constant. Both phases are assumed compressible with mass densities denoted by $\rho^{(\alpha)}(p), \alpha=1,2$. The rock permeability is denoted by $\mathbf{K}$ and the rock porosity by $\phi$. Then, the pressure $p$ and saturations $s^{(\alpha)}, \alpha=1,2$ unknowns are solutions of the mass conservation of the gas and liquid phases with phase velocities given by the Darcy laws, coupled to the pore volume conservation:

$$
\left\{\begin{aligned}
\partial_{t}\left(\phi \rho^{(\alpha)}(p) s^{(\alpha)}\right)+\operatorname{div}\left(\rho^{(\alpha)}(p) \mathbf{V}^{(\alpha)}\right)=0, \alpha=1,2, & \text { on } \Omega_{r} \times(0, T), \\
s^{(1)}+s^{(2)}=1, & \text { on } \Omega_{r} \times(0, T), \\
-\mathbf{K}\left(\nabla p-\rho^{(\alpha)}(p) \mathbf{g}\right) \cdot \mathbf{n}=0, \alpha=1,2, & \text { on } \Gamma_{r} \times(0, T), \\
p=p_{\text {inj }}(z), s^{(\alpha)}=s_{\text {inj }}^{(\alpha)}, \alpha=1,2, & \text { on } \Gamma_{w} \times(0, T), \\
p=p_{\text {init }}(z), s^{(\alpha)}=s_{\text {init }}^{(\alpha)}, \alpha=1,2, & \text { on } \Omega_{r} \times\{0\} .
\end{aligned}\right.
$$

We have used the following additional notations: $s_{\text {init }}^{(1)}=1, s_{\text {init }}^{(2)}=0$ are the initial saturations, $p_{\text {init }}(z)$ is the initial hydrostatic phase 1 pressure such that

$$
\frac{d p_{\text {init }}(z)}{d z}=-\rho^{(1)}\left(p_{\text {init }}(z)\right) g, p_{\text {init }}\left(z_{\text {ref }}\right)=\bar{p}_{\text {init }}
$$

$s_{\text {inj }}^{(1)}=0, s_{\text {inj }}^{(2)}=1$ are the saturations at the injection well and $p_{\text {inj }}(z)$ is the injection well imposed pressure such that

$$
\frac{d p_{\text {inj }}(z)}{d z}=-\rho^{(2)}\left(p_{\text {inj }}(z)\right) g, p_{\text {inj }}\left(z_{r e f}\right)=p_{b h p}
$$

where $p_{b h p}$ is the prescribed bottom hole pressure at the reference height $z_{\text {ref }}$. It is assumed that the bottom hole pressure $p_{b h p}$ and the initial pressure $\bar{p}_{\text {init }}$ are such that $-\mathbf{K}\left(\nabla p-\rho^{(\alpha)} \mathbf{g}\right) \cdot \mathbf{n}_{w}<0$ for $\alpha=1,2$ at the well 
boundary $\Gamma_{w}$, where $\mathbf{n}_{w}$ is the unit normal vector at the well boundary outward to $\Omega_{r}$. The case of a producer well could also be dealt without additional difficulties as it will be the case in the numerical experiments.

Due to the impervious outer boundary of the reservoir, and the small compressibility of the liquid phase, the gas front will be in practice localized in a small region around the vertical well which motivates the use of nearwell local mesh and time refinement for the simulation of this model. Let us denote by $\Omega_{w} \subset \Omega_{r}$ a nearwell region which is chosen such that the gas phase will mostly remain in $\Omega_{w}$ during the gas injection. In the following, the outer boundary of the nearwell region $\Omega_{w}$ will be denoted by $\Gamma_{r w}$ (see figure 1).

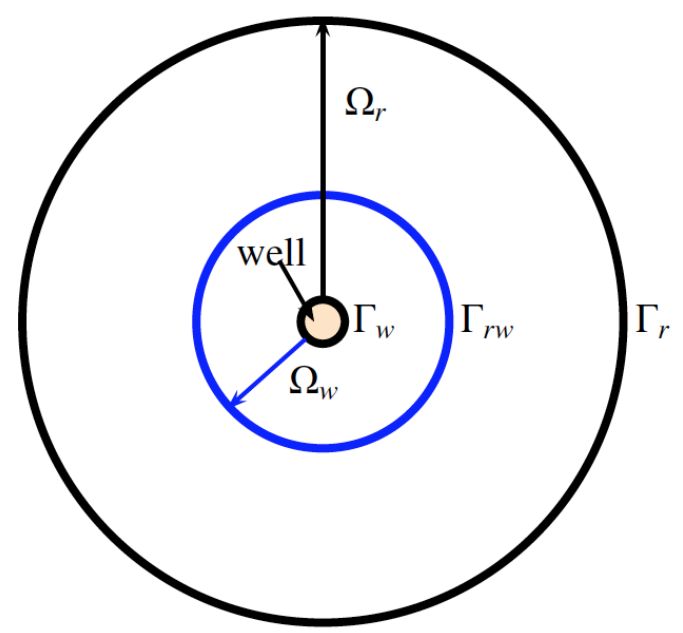

FiguRE 1. Example of reservoir domain $\Omega_{r}$ and nearwell subdomain $\Omega_{w}$ with the reservoir nearwell interface $\Gamma_{r w}$, and the well boundaries $\Gamma_{w}$.

A DDM method will be used to solve the two phase flow model with a coarse discretization in space and time on the reservoir domain $\Omega_{r}$ and a locally refined space and time discretization on the nearwell region $\Omega_{w}$. The coupling between both discretizations will be obtained by solving iteratively both subproblems on a given time interval $\left(t^{n-1}, t^{n}\right)$ using appropriate interface conditions at $\Gamma_{w}$ and $\Gamma_{r w}$. A Robin condition for the pressure together with an input Dirichlet condition for the saturations will be used at the boundary $\Gamma_{r w}$ of the subdomain $\Omega_{w}$. At the well boundary $\Gamma_{w}$ of the domain $\Omega_{r}$, a total flux Neumann condition together with an input Dirichlet condition for the saturations will be imposed.

In the following subsections, the coarse and fine finite volume discretizations of $\Omega_{r}$ and $\Omega_{w}$ are first introduced, then the DDM algorithm is described with a single time stepping, and finally the extension to take into account LTS in the nearwell domain is explained.

\subsection{Two level Finite Volume discretization}

As exhibited in figure 2, the discretization starts from a coarse finite volume discretization of the full reservoir domain $\Omega_{r}$ defined by

$$
\left(\mathcal{M}_{r}, \mathcal{F}_{r}^{\text {int }}, \mathcal{P}_{r}\right)
$$

where $\mathcal{M}_{r}$ is the set of coarse cells $K, \mathcal{F}_{r}^{\text {int }}$ the set of coarse inner faces $\sigma$, and $\mathcal{P}_{r}$ the set of coarse well perforations. The mesh is assumed to be conforming in the sense that the set of neighbouring cells $\mathcal{M}_{\sigma} \subset \mathcal{M}_{r}$ of an inner face $\sigma \in \mathcal{F}_{r}^{\text {int }}$ contains exactly two cells $K$ and $L$. The inner face $\sigma$ will also be denoted by $\sigma=K \mid L$. Considering that the size of the cells is very large compared with the well radius, the wells are as usual discretized using Peaceman's indices in each perforated cell [11]. For each perforation $\sigma \in \mathcal{P}_{r}$, let us denote by $K_{\sigma}^{r} \in \mathcal{M}_{r}$ the corresponding perforated coarse cell.

A set of nearwell coarse cells is assumed to be refined (coarse cells inside the red boundary in figure 2) and the nearwell mesh is obtained by addition of one layer of coarse cells at the boundary of the union of the refined cells. The resulting nearwell mesh is defined by

$$
\left(\mathcal{M}_{w}, \mathcal{F}_{w}^{\mathrm{int}}, \mathcal{F}_{r w}, \mathcal{P}_{w}\right)
$$


where $\mathcal{M}_{w}$ is the set of cells $K, \mathcal{F}_{w}^{\text {int }}$ is the set of inner faces $\sigma, \mathcal{P}_{w}$ is the set of fine well perforations, and $\mathcal{F}_{r w} \subset \mathcal{F}_{r}^{\text {int }}$ is the set of boundary faces corresponding by construction to coarse faces. The fine perforated cells will be denoted by $K_{\sigma}^{w}$ for all perforations $\sigma \in \mathcal{P}_{w}$ as exhibited in figure 2. It is again assumed that the nearwell mesh is conforming in the sense that the set of neighbouring cells $\mathcal{M}_{\sigma} \subset \mathcal{M}_{w}$ of an inner face $\sigma \in \mathcal{F}_{w}^{\text {int }}$ contains exactly two cells $K$ and $L$, and the inner face $\sigma$ will also be denoted by $\sigma=K \mid L$. At the nearwell reservoir interface, for each face $\sigma \in \mathcal{F}_{r w}$, it is assumed that the set of the two neighbouring cells $\mathcal{M}_{\sigma}=\{K, L\}$ is ordered such that $K \in \mathcal{M}_{w} \cap \mathcal{M}_{r}$ and $L \in \mathcal{M}_{r} \backslash \mathcal{M}_{w}$.

A cell centre finite volume discretization is used for the discretization of the two phase flow model. We will denote by $P_{r}$ (resp. $P_{w}$ ) the vector of cell pressures $P_{r, K}, K \in \mathcal{M}_{r}$ (resp. $P_{w, K}, K \in \mathcal{M}_{w}$ ) and similarly by $S_{r}^{(\alpha)}$ (resp. $\left.S_{w}^{(\alpha)}\right)$ the vector of cell saturations $S_{r, K}^{(\alpha)}, K \in \mathcal{M}_{r}\left(\operatorname{resp} . S_{w, K}^{(\alpha)}, K \in \mathcal{M}_{w}\right)$ with $\alpha=1,2$.

Let $\sigma=K \mid L$ be an inner coarse or fine face, and $\mathbf{n}_{K, \sigma}$ the unit normal vector at the face $\sigma$ outward to the cell $K$. Let $P$ be the reservoir or nearwell discrete pressure $P_{r}$ or $P_{w}$. Assuming the orthogonality of the mesh w.r.t. the permeability field $\mathbf{K}$, the Darcy flux

$$
\int_{\sigma}-\mathbf{K}\left(\nabla p-\rho^{(\alpha)}(p) \mathbf{g}\right) \cdot \mathbf{n}_{K, \sigma} d \sigma
$$

is approximated by the following conservative Two Point Flux Approximation (TPFA) [6]

$$
F_{K, \sigma}^{(\alpha)}(P)=T_{\sigma}\left(P_{K}-P_{L}+\rho^{(\alpha)}\left(\frac{P_{K}+P_{L}}{2}\right) g\left(z_{K}-z_{L}\right)\right), \alpha=1,2
$$

where $T_{\sigma}$ is the transmissivity of the face $\sigma \in \mathcal{F}_{r}^{\text {int }}$ or $\sigma \in \mathcal{F}_{w}^{\text {int }}$, and $z_{K}, z_{L}$ are the $z$ coordinates of the centres of the cells $K, L$.

A Two Point flux approximation of the Darcy flux is also assumed at the nearwell reservoir interface $\sigma=$ $K \mid L \in \mathcal{F}_{r w}$. It is denoted by

$$
F_{K, \sigma}^{(\alpha)}\left(P_{w, K}, P_{r, L}\right)=T_{\sigma}\left(P_{w, K}-P_{r, L}+\rho^{(\alpha)}\left(\frac{P_{w, K}+P_{r, L}}{2}\right) g\left(z_{K}-z_{L}\right)\right), \alpha=1,2
$$

where $T_{\sigma}$ is the transmissivity of the face $\sigma$, and $z_{K}, z_{L}$ are the $z$ coordinates of the centres of the cells $K, L$. It is to be understood that, in the following DDM algorithm, $P_{r, L}$ will represent the pressure interface value viewed by the nearwell subdomain. In other words, we have chosen to discretize the boundary conditions at the reservoir nearwell interface using cell values for the interface pressures and face values for the fluxes in order to obtain the same finite volume discretization than the one obtained on the single LGR mesh $\mathcal{M}^{\text {lgr }}$ exhibited in figure 3 (see [7] for a discussion on the choice of cell values rather than face values for the interface pressures).

The extension of the Darcy fluxes discretization to Multi Point Flux Approximations (MPFA, see for example [2]) is straightforward and involves, on the nearwell side, MPFA Darcy fluxes depending both on the nearwell pressure $P_{w, K}$ and on the reservoir pressures $P_{r, L}$ for all $K \in \mathcal{M}_{w}$ and $L \in \mathcal{M}_{r}$ such that $K \mid L \in \mathcal{F}_{r w}$.

For each $\sigma \in \mathcal{P}_{s}, s=r, w$, the approximation of the Darcy fluxes $\int_{\sigma}-\mathbf{K}\left(\nabla p-\rho^{\alpha}(p) \mathbf{g}\right) \cdot \mathbf{n}_{K, \sigma} d \sigma, \alpha=1,2$, at the well perforation boundary is defined by the two point flux approximation

$$
F_{K, \sigma}^{s}\left(P_{s, K}, P_{\sigma}\right)=P I_{\sigma}^{s}\left(P_{s, K}-P_{\sigma}\right)
$$

where $P_{\sigma}$ denotes the pressure in the perforation $\sigma, K=K_{\sigma}^{s}$ is the coarse $(s=r)$ or fine $(s=w)$ perforated cell, and $P I_{\sigma}^{s}$ for $s=r$ or $s=w$ is the transmissivity of the perforation $\sigma$ in the cell $K$ obtained using the Peaceman formula which takes into account the singularity of the pressure solution at the well (see [11]). Note that it has been assumed that the $\mathrm{z}$ coordinate of the centre of the perforation $z_{\sigma}$ matches with the $\mathrm{z}$ coordinate of the cell centre $z_{K}$. 

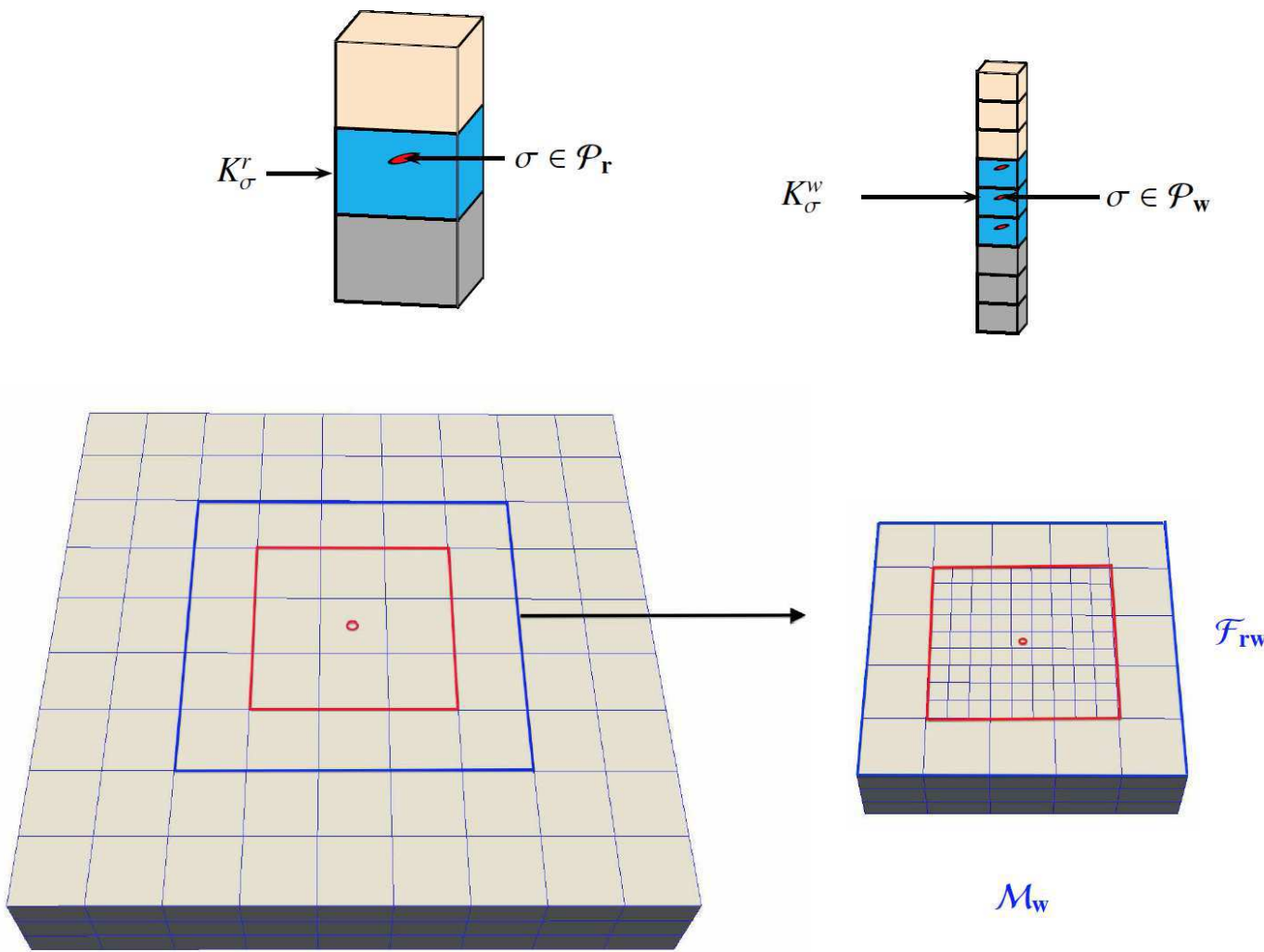

$\mathcal{M}_{\mathrm{w}}$

$\mathcal{M}_{r}$

Figure 2. Bottom left: reservoir coarse mesh $\mathcal{M}_{r}$. Bottom right: nearwell fine mesh $\mathcal{M}_{w}$ and reservoir nearwell interfaces $\mathcal{F}_{r w}$. Top left: coarse cell $K_{\sigma}^{r}$ of the perforation $\sigma \in \mathcal{P}_{r}$. Top right: fine cell $K_{\sigma}^{w}$ of the perforation $\sigma \in \mathcal{P}_{w}$.

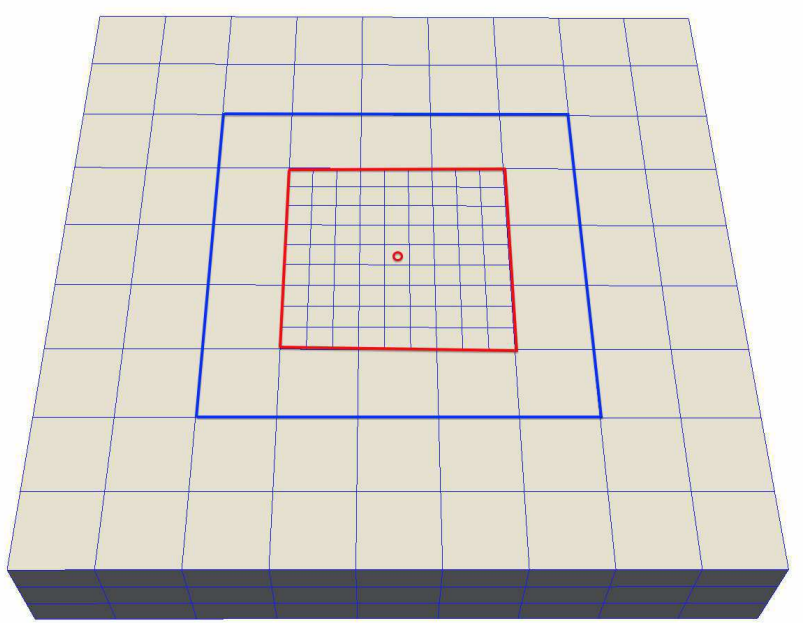

FIGURE 3. LGR mesh $\mathcal{M}^{\text {lgr }}$ of the full reservoir domain. 


\subsection{Optimized Schwarz Domain Decomposition method with full overlap at the coarse level}

The idea behind our choice of the interface conditions at the reservoir nearwell interface $\Gamma_{r w}$ is to uncouple the pressure and the saturations in the definitions of the interface conditions in such a way that the DDM algorithm can be easily generalized to multiphase compositional Darcy flow models, and will be easier to implement in existing reservoir simulators. It leads to the following choice of the interface conditions at the interface $\Gamma_{r w}$ :

$$
\left\{\begin{array}{l}
\lambda p_{w}+\alpha \mathbf{K} \nabla p_{w} \cdot \mathbf{n}_{w}=\lambda p_{r}+\alpha \mathbf{K} \nabla p_{r} \cdot \mathbf{n}_{w} \\
s_{w}^{(\alpha)}=s_{r}^{(\alpha)}, \alpha=1,2, \text { if }-\mathbf{K}\left(\nabla p_{w}-\rho^{(\alpha)}\left(p_{w}\right) \mathbf{g}\right) \cdot \mathbf{n}_{w}<0
\end{array}\right.
$$

where $\mathbf{n}_{w}$ is the normal at $\Gamma_{r w}$ outward $\Omega_{w}$ and $\alpha, \lambda$ are positive parameters defined below. At the well boundary $\Gamma_{w}$, a Neumann total flux condition will be used since it is a usual monitoring condition for wells in reservoir simulators. In our example of an injection well we obtain the following condition

$$
\left\{\begin{array}{l}
p_{r}=p_{\mathrm{r}, \mathrm{inj}}(z), \\
s_{r}^{(\alpha)}=s_{\mathrm{inj}}^{(\alpha)}, \alpha=1,2 \\
\frac{d p_{\mathrm{r}, \mathrm{inj}}(z)}{d z}=-\rho^{(2)}\left(p_{\mathrm{r}, \mathrm{inj}}(z)\right) g, p_{\mathrm{r}, \mathrm{inj}}\left(z_{r e f}\right)=p_{r, b h p}, \\
\sum_{\alpha=1,2} \int_{\Gamma_{w}}-\rho^{(\alpha)}\left(p_{r}\right) \frac{k_{r}^{(\alpha)}\left(s_{r}^{(\alpha)}\right)}{\mu^{(\alpha)}} \mathbf{K} \nabla p_{r} \cdot \mathbf{n} d \sigma=\sum_{\alpha=1,2} \int_{\Gamma_{w}}-\rho^{(\alpha)}\left(p_{\mathrm{inj}}(z)\right) \frac{k_{r}^{(\alpha)}\left(s_{\mathrm{inj}}^{(\alpha)}\right)}{\mu^{(\alpha)}} \mathbf{K} \nabla p_{w} \cdot \mathbf{n} d \sigma,
\end{array}\right.
$$

where the coarse bottom hole pressure $p_{r, b h p}$ is the additional unknown corresponding to the total flux additional equation.

As stated above, these interface conditions are discretized using cell values for the interface pressures or saturations and face values for the fluxes in order to obtain the same finite volume discretization than on the LGR grid exhibited in figure 3 .

Let us consider, on both the reservoir and nearwell meshes, the same time discretization $t^{0}, t^{1}, \cdots, t^{N}$ of the interval $(0, T)$ with $t^{0}=0, t^{N}=T$, and $\Delta t^{n}=t^{n}-t^{n-1}>0, n=1, \cdots, N$. The two phase flow model is integrated by an implicit Euler scheme coupling implicitly the pore volume conservation and the mass conservation of both phases. The discretization in space uses the TPFA discretization of the Darcy fluxes together with an upwinding of the relative permeabilities with respect to the sign of the Darcy fluxes (see [3], [11]).

Let the reservoir and nearwell solutions at time $t^{n-1}$ be given. In the following, the pressure $P$ and the saturation $S^{(2)}$ are chosen as the primary unknowns and it is always implicitly assumed that the liquid saturation $S^{(1)}$ is defined by $S^{(1)}=1-S^{(2)}$. Then, knowing the nearwell solution $P_{w}, S_{w}^{(2)}$, the reservoir subproblem computes the solution $P_{r}, S_{r}^{(2)}, P_{r, b h p}$ of the following conservation equations in each cell $K \in \mathcal{M}_{r}$ for both phases $\alpha=1,2$

$$
\left\{\begin{array}{l}
\phi_{K}\left(\rho^{(\alpha)}\left(P_{r, K}\right) S_{r, K}^{(\alpha)}-\rho^{(\alpha)}\left(P_{r, K}^{n-1}\right) S_{r, K}^{(\alpha), n-1}\right) \frac{|K|}{\Delta t^{n}} \\
+\sum_{\sigma=K \mid L \in \mathcal{F}_{r}^{\text {int }}} \frac{\rho^{(\alpha)}\left(P_{r, K}\right) k_{r}^{(\alpha)}\left(S_{r, K}^{(\alpha)}\right)}{\mu^{(\alpha)}} F_{K, \sigma}^{(\alpha)}\left(P_{r}\right)^{+}+\sum_{\sigma=K \mid L \in \mathcal{F}_{r}^{\text {int }}} \frac{\rho^{(\alpha)}\left(P_{r, L}\right) k_{r}^{(\alpha)}\left(S_{r, L}^{(\alpha)}\right)}{\mu^{(\alpha)}} F_{K, \sigma}^{(\alpha)}\left(P_{r}\right)^{-} \\
+\sum_{\sigma \in \mathcal{P}_{r} \mid K_{\sigma}^{r}=K} \frac{\rho^{(\alpha)}\left(P_{r, \sigma}\right) k_{r}^{(\alpha)}\left(S_{r, \sigma}^{(\alpha)}\right)}{\mu^{(\alpha)}} F_{K, \sigma}^{r}\left(P_{r, K}, P_{r, \sigma}\right)=0,
\end{array}\right.
$$


coupled with the well total flux condition

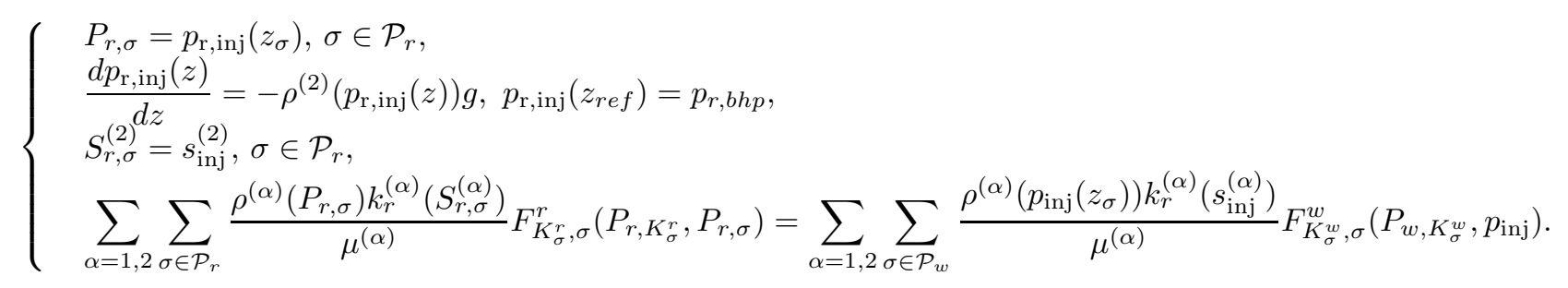

Similarly, knowing the reservoir solution $P_{r}, S_{r}^{(2)}$, the nearwell subproblem computes the solution $P_{w}, S_{w}^{(2)}$ of the conservation equations in each cell $K \in \mathcal{M}_{w}$ for both phases $\alpha=1,2$

$$
\left\{\begin{array}{l}
\phi_{K}\left(\rho^{(\alpha)}\left(P_{w, K}\right) S_{w, K}^{(\alpha)}-\rho^{(\alpha)}\left(P_{w, K}^{n-1}\right) S_{w, K}^{(\alpha), n-1}\right) \frac{|K|}{\Delta t^{n}} \\
+\sum_{\sigma=K \mid L \in \mathcal{F}_{w}^{\mathrm{int}}} \frac{\rho^{(\alpha)}\left(P_{w, K}\right) k_{r}^{(\alpha)}\left(S_{w, K}^{(\alpha)}\right)}{\mu^{(\alpha)}} F_{K, \sigma}^{(\alpha)}\left(P_{w}\right)^{+}+\sum_{\sigma=K \mid L \in \mathcal{F}_{w}^{\mathrm{int}}} \frac{\rho^{(\alpha)}\left(P_{w, L}\right) k_{r}^{(\alpha)}\left(S_{w, L}^{(\alpha)}\right)}{\mu^{(\alpha)}} F_{K, \sigma}^{(\alpha)}\left(P_{w}\right)^{-} \\
+\sum_{\sigma=K \mid L \in \mathcal{F}_{r w}} \frac{\rho^{(\alpha)}\left(P_{w, K}\right) k_{r}^{(\alpha)}\left(S_{w, K}^{(\alpha)}\right)}{\mu^{(\alpha)}} F_{K, \sigma}^{(\alpha)}\left(P_{w, K}, P_{w, \sigma}\right)^{+}+\sum_{\sigma=K \mid L \in \mathcal{F}_{r w}} \frac{\rho^{(\alpha)}\left(P_{w, \sigma}\right) k_{r}^{(\alpha)}\left(S_{w, \sigma}^{(\alpha)}\right)}{\mu^{(\alpha)}} F_{K, \sigma}^{(\alpha)}\left(P_{w, K}, P_{w, \sigma}\right)^{-} \\
+\sum_{\sigma \in \mathcal{P}_{w} \mid K_{\sigma}^{w}=K} \frac{\rho^{(\alpha)}\left(p_{\mathrm{inj}}\right) k_{r}^{(\alpha)}\left(s_{\mathrm{inj}}^{(\alpha)}\right)}{\mu^{(\alpha)}} F_{K, \sigma}^{w}\left(P_{w, K}, p_{\mathrm{inj}}\left(z_{\sigma}\right)\right)=0,
\end{array}\right.
$$

coupled with the following reservoir nearwell interface conditions for all $\sigma=K \mid L \in \mathcal{F}_{r w}$

$$
\left\{\begin{array}{l}
|\sigma| \lambda_{\sigma} P_{w, \sigma}-\alpha_{\sigma} F_{K, \sigma}\left(P_{w, K}, P_{w, \sigma}\right) \\
=|\sigma| \lambda_{\sigma} P_{r, L}-\alpha_{\sigma} F_{K, \sigma}\left(P_{r, K}, P_{r, L}\right) \\
S_{w, \sigma}^{(2)}=S_{r, L}^{(2)}
\end{array}\right.
$$

In the above equations $\phi_{K}$ denotes the porosity, and $|K|$ the volume of the cell $K,|\sigma|$ the surface of the face $\sigma$, and $x^{+}=\max (x, 0), x^{-}=\min (x, 0)$.

Let us set

and

$$
P_{w, \mathcal{F}_{r w}}=\left(P_{w, \sigma}, \sigma \in \mathcal{F}_{r w}\right), \quad S_{w, \mathcal{F}_{r w}}^{(2)}=\left(S_{w, \sigma}^{(2)}, \sigma \in \mathcal{F}_{r w}\right),
$$

$$
P_{r, \mathcal{F}_{r w}}=\left(P_{r, L}, \mathcal{M}_{\sigma}=K \mid L, \sigma \in \mathcal{F}_{r w}\right), \quad S_{r, \mathcal{F}_{r w}}^{(2)}=\left(S_{r, L}^{(2)}, \mathcal{M}_{\sigma}=K \mid L, \sigma \in \mathcal{F}_{r w}\right) .
$$

Using these notations, we can rewrite the reservoir subproblem (4)-(5) as follows

$$
\left\{\begin{aligned}
\mathcal{R}_{r}\left(P_{r}, S_{r}^{(2)}, p_{\mathrm{r}, \mathrm{bhp}}\right) & =0 \\
\mathcal{B}_{Q_{T}}\left(P_{r}, p_{\mathrm{r}, \mathrm{bhp}}, s_{\mathrm{inj}}^{(2)}\right) & =\mathcal{B}_{Q_{T}}\left(P_{w}, p_{\mathrm{bhp}}, s_{\mathrm{inj}}^{(2)}\right),
\end{aligned}\right.
$$

where $\mathcal{R}_{r}$ denotes the system of reservoir conservation equations, and $\mathcal{B}_{Q_{T}}$ denotes the well total flux condition. Similarly, we can rewrite the nearwell subproblem (6)-(7) as follows

$$
\left\{\begin{aligned}
\mathcal{R}_{w}\left(P_{w}, S_{w}^{(2)}, P_{w, \mathcal{F}_{r w}}, S_{w, \mathcal{F}_{r w}}^{(2)}\right) & =0 \\
\mathcal{B}_{\text {robin }}\left(P_{w}, P_{w, \mathcal{F}_{r w}}\right) & =\mathcal{B}_{\text {robin }}\left(P_{r}, P_{r, \mathcal{F}_{r w}}\right), \\
S_{w, \mathcal{F}_{r w}}^{(2)} & =S_{r, \mathcal{F}_{r w}}^{(2)}
\end{aligned}\right.
$$

where $\mathcal{R}_{w}$ denotes the system of reservoir conservation equations, and $\mathcal{B}_{\text {robin }}$ denotes the Robin boundary condition for the pressure at the interface $\Gamma_{r w}$. 
Then, the DDM algorithm, at the given time step $\Delta t^{n}$, is the following multiplicative Schwarz algorithm which computes the reservoir and nearwell solutions $\left(P_{r}, S_{r}^{(2)}\right)$, and $\left(P_{w}, S_{w}^{(2)}\right)$ of the coupled systems (4)-(5)-(6)-(7) solving successively the following subproblems

$$
\begin{aligned}
& \left\{\begin{array}{l}
\mathcal{R}_{r}\left(P_{r}^{k}, S_{r}^{(2), k}, p_{\mathrm{r}, \mathrm{bhp}}^{k}\right)=0, \\
\mathcal{B}_{Q_{T}}\left(P_{r}^{k}, p_{\mathrm{r}, \mathrm{bhp}}^{k}, s_{\mathrm{inj}}^{(2)}\right)=\mathcal{B}_{Q_{T}}\left(P_{w}^{k-1}, p_{\mathrm{bhp}}, s_{\mathrm{inj}}^{(2)}\right),
\end{array}\right. \\
& \left\{\begin{aligned}
\mathcal{R}_{w}\left(P_{w}^{k}, S_{w}^{(2), k}, P_{w, \mathcal{F}_{r w}}^{k}, S_{w, \mathcal{F}_{r w}}^{(2), k}\right) & =0, \\
\mathcal{B}_{\text {robin }}\left(P_{w}^{k}, P_{w, \mathcal{F}_{r w}}^{k}\right) & =\mathcal{B}_{\text {robin }}\left(P_{r}^{k}, P_{r, \mathcal{F}_{r w}}^{k}\right), \\
S_{w, \mathcal{F}_{r w}}^{(2), k} & =S_{r, \mathcal{F}_{r w}}^{(2), k},
\end{aligned}\right.
\end{aligned}
$$

for $k \geq 1$ until the following stopping criteria is fulfilled:

$$
d Q=\frac{\left|\mathcal{B}_{Q_{T}}\left(P_{w}^{k}, p_{\mathrm{bhp}}, s_{\mathrm{inj}}^{(2)}\right)-\mathcal{B}_{Q_{T}}\left(P_{w}^{k-1}, p_{\mathrm{bhp}}, s_{\mathrm{inj}}^{(2)}\right)\right|}{\left|\mathcal{B}_{Q_{T}}\left(P_{w}^{k}, p_{\mathrm{bhp}}, s_{\mathrm{inj}}^{(2)}\right)\right|} \leq \epsilon,
$$

for a given $\epsilon$. Note that, due to the non matching overlap of reservoir and nearwell meshes, the solution depends on the parameters $\alpha$ and $\lambda$ and will not a priori match with the solution obtained on the LGR mesh $\mathcal{M}^{\text {lgr }}$. Let us also remark that the Neumann total flux condition at the well for the coarse mesh system is replaced by the pressure Dirichlet condition $p_{\text {inj }}$ at the first iteration at least at the first time step.

The parameters $\alpha_{\sigma}$ are set to 1 and the parameters $\lambda_{\sigma}>0, \sigma \in \mathcal{F}_{r w}$ are chosen to optimize the convergence rate leading to an optimized Robin - Neumann DDM algorithm. Let us refer to [8] for a detailed discussion on optimized Schwarz methods for diffusion problems including optimized Robin interface conditions. Our application of this existing theory to two phase flows is based on the idea that it suffices to optimize the parameter $\lambda$ for a fixed linearized parabolic pressure equation in the spirit of uncoupling the interface conditions for the pressure and for the saturations. In our case, the optimization of $\lambda$ will be typically based on the following liquid phase pressure equation

$$
c^{l} \partial_{t} p+\operatorname{div}\left(-\frac{\mathbf{K}}{\mu^{(1)}} \nabla p\right)=0
$$

where $c^{l}$ is the compressibility of the liquid phase. We refer to the numerical tests section below for an example of computation of the optimized Robin coefficients based on such parabolic equation.

\subsection{Local Time Stepping}

Let $t^{0}, t^{1}, \cdots, t^{N}$ denote the coarse time discretization on the reservoir domain with the coarse time stepping $\Delta t^{n}=t^{n}-t^{n-1}>0, n=1, \cdots, N$. Each time interval $\left(t^{n-1}, t^{n}\right)$ is discretized using a local time stepping on the nearwell subdomain denoted by $t^{n, m}, m=0, \cdots, N_{n}$ with $\Delta t^{n, m}=t^{n, m}-t^{n, m-1}>0$ for all $m=1, \cdots, N_{n}$, and $t^{n, 0}=t^{n-1}, t^{n, N_{n}}=t^{n}$ (see figure 4 ).

The extension of the previous DDM algorithm to local time stepping follows the ideas of [5] for parabolic problems with some adaptations to the case of two phase flows. Firstly, the boundary conditions at the reservoir nearwell interface are interpolated in time between the two successive coarse times $t^{n-1}$ and $t^{n}$ :

$$
\left\{\begin{aligned}
\mathcal{B}_{\text {robin }}\left(P_{w}^{n, m, k}, P_{w, \mathcal{F}_{r w}}^{n, m, k}\right)= & \frac{t^{n, m}-t^{n-1}}{\Delta t^{n}} \mathcal{B}_{\text {robin }}\left(P_{r}^{k, n}, P_{r, \mathcal{F}_{r w}}^{k, n}\right) \\
& +\frac{t^{n}-t^{n, m}}{\Delta t^{n}} \mathcal{B}_{\text {robin }}\left(P_{r}^{k, n-1}, P_{r, \mathcal{F}_{r w}}^{k, n-1}\right) \\
S_{w, \mathcal{F}_{r w}}^{(2), k, n, m}= & \frac{t^{n, m}-t^{n-1}}{\Delta t^{n}} S_{r, \mathcal{F}_{r w}}^{(2), k, n}+\frac{t^{n}-t^{n, m}}{\Delta t^{n}} S_{r, \mathcal{F}_{r w}}^{(2), k, n-1} .
\end{aligned}\right.
$$

Secondly, at each well perforation of the reservoir coarse mesh, the time average of the total flux between $t^{n-1}$ and $t^{n}$ is imposed:

$$
\mathcal{B}_{Q_{T}}\left(P_{r}^{n, k}, p_{\mathrm{r}, \mathrm{bhp}}^{n, k}, s_{\text {inj }}^{(2)}\right)=\sum_{m=1}^{N_{n}} \frac{\Delta t^{n, m}}{\Delta t^{n}} \mathcal{B}_{Q_{T}}\left(P_{w}^{n, m, k-1}, p_{\text {bhp }}, s_{\text {inj }}^{(2)}\right) .
$$

In order to improve the time discretization of the algorithm, we also use a $\theta$ scheme for the time integration in the coarse reservoir mesh with $\theta=1$ (implicit Euler) at the first coarse time step $\left(t^{0}, t^{1}\right)$ to account for the steep gradient induced by the nearly incompressible liquid phase, and with $\theta=\frac{3}{5}$ for the remaining coarse time 
steps. The $\theta$ scheme is only applied on the pressure unknown, and the implicit Euler scheme is kept on the saturation unknowns.

The choice of the coarse time discretization plays a crutial role in the accuracy of the algorithm. It should be done in practice adaptively in order to control the maximum variation of the pressure and of the saturations at the reservoir nearwell interface during a coarse time step. This can be easily computed using the first coarse reservoir solution of the DDM iterations to adapt the coarse time stepping.

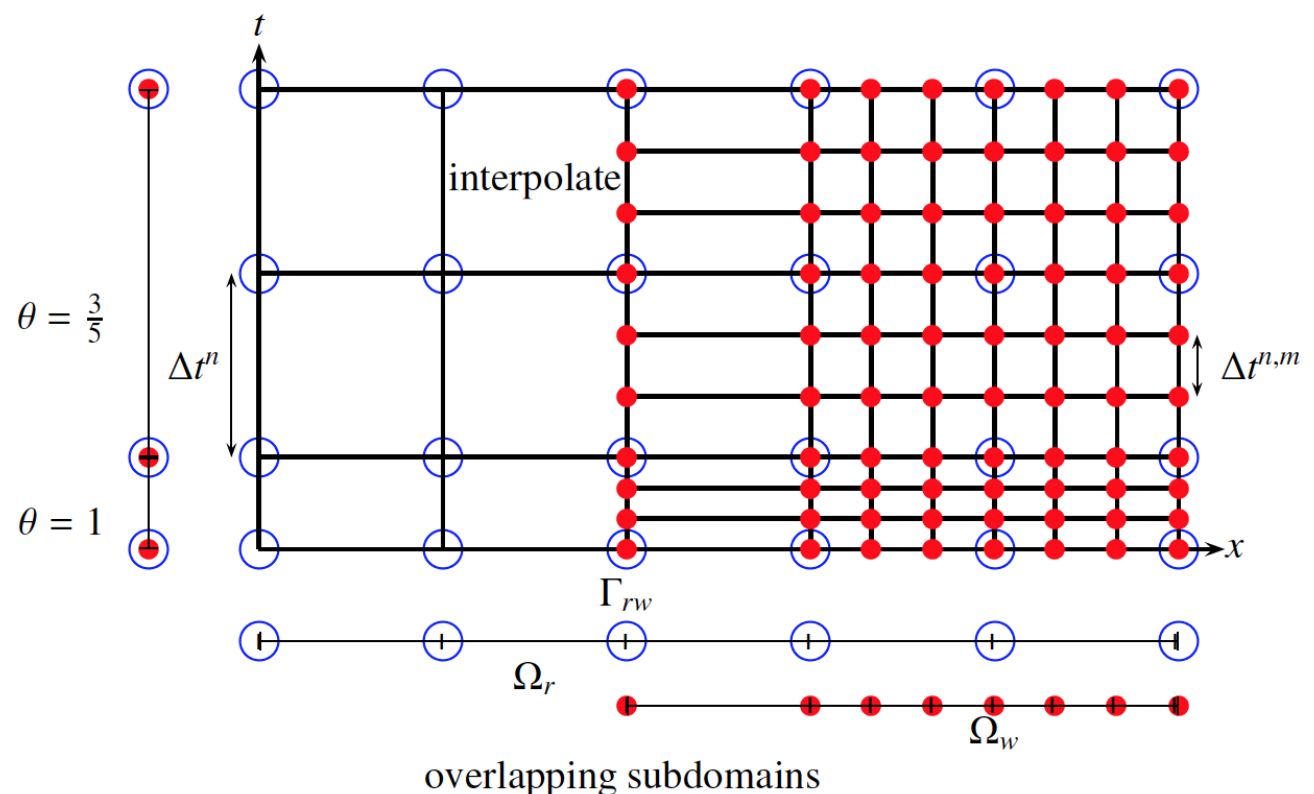

Figure 4. Time-space discretization.

\section{NUMERICAL TESTS}

In this section our algorithms are compared with the classical Windowing algorithm on two 3D test cases using a Black Oil type model [12]. A reference solution is obtained using the LGR grid with the global fine time stepping. The model used in both test cases describes the Darcy flow of two phases denoted by $\alpha=1,2$ where phase 1 contains two components $i=1,2$ and phase 2 contains only the component 2 . Phase 1 is always assumed to be present, and we denote by $c_{2}^{(1)}$ the mass fraction of component 2 in phase 1 . Taking into account capillary effects, the pressures of both phases are denoted by $p^{(\alpha)}, \alpha=1,2$ and connected by the capillary pressure function $p_{c}$ such that $p_{c}\left(s^{(2)}\right)=p^{(2)}-p^{(1)}$. If both phases are present, component 1 is assumed to be at thermodynamical equilibrium characterized by the equation $c_{2}^{(1)}=f\left(p^{(2)}\right)$, while the inequality $c_{2}^{(1)} \leq f\left(p^{(2)}\right)$ is imposed if only phase 1 is present. The velocities of the phases are modified to take into account the capillary pressure as follows

$$
\mathbf{V}^{(\alpha)}=-\frac{k_{r}^{(\alpha)}\left(s^{(\alpha)}\right)}{\mu^{(\alpha)}} \mathbf{K}\left(\nabla p^{(\alpha)}-\rho^{(\alpha)} \mathbf{g}\right), \alpha=1,2,
$$

Using the Coat's formulation [4], we obtain the following system of equations for the primary unknowns $s^{(\alpha)}, p^{(\alpha)}, \alpha=1,2$ and $c_{2}^{(1)}$ :

$$
\left\{\begin{array}{rr}
\partial_{t}\left(\phi \rho^{(1)}\left(p^{(1)}\right) s^{(1)}\left(1-c_{2}^{(1)}\right)+\operatorname{div}\left(\rho^{(1)}\left(p^{(1)}\right)\left(1-c_{2}^{(1)}\right) \mathbf{V}^{(1)}\right)=0,\right. & \text { on } \Omega_{r} \times(0, T), \\
\partial_{t}\left(\phi \rho^{(1)}\left(p^{(1)}\right) s^{(1)} c_{2}^{(1)}+\phi \rho^{(2)}\left(p^{(2)}\right) s^{(2)}\right)+\operatorname{div}\left(\rho^{(1)}\left(p^{(1)}\right) c_{2}^{(1)} \mathbf{V}^{(1)}+\rho^{(2)}\left(p^{(2)}\right) \mathbf{V}^{(2)}\right)=0, & \text { on } \Omega_{r} \times(0, T), \\
s^{(2)}+s^{(1)}=1, & \text { on } \Omega_{r} \times(0, T), \\
p^{(2)}-p^{(1)}=p_{c}\left(s^{(2)}\right), & \text { on } \Omega_{r} \times(0, T), \\
s^{(2)}\left(c_{2}^{(1)}-f\left(p^{(2)}\right)\right)=0, & \text { on } \Omega_{r} \times(0, T), \\
c_{2}^{(1)} \leq f\left(p^{(2)}\right), \quad s^{(2)} \geq 0, & \text { on } \Omega_{r} \times(0, T) .
\end{array}\right.
$$


In both test cases, a multi-perforated vertical well with either injection or production is considered at the centre of the domain with imposed bottom hole pressure $p_{b h p}$.

Our Robin-Neumann DDM algorithm easily extends to such a Black Oil model. At the reservoir nearwell interface, input Dirichlet boundary conditions for $S^{(\alpha)}, \alpha=1,2$ and $c_{2}^{(1)}$ are used, and a Robin optimized condition is used for the reference pressure chosen to be the phase 1 pressure $p^{(1)}$. The optimization of the Robin coefficient will be based on a 1D linear parabolic equation for $p^{(1)}$ leading to a simple analytical formula (see below). At the multi-perforated well, a total flux condition is imposed on the reservoir coarse well obtained from the nearwell fine model by summing the mass fluxes contributions of both components 1 and 2 and of all perforations of the fine well.

In both test cases, the reservoir domain $\Omega_{r}$ is defined by a spherical cap of vertical axis $x=y=0$, of horizontal projection $(-L, L) \times(-L, L)$, of width $H$, and of bending $b$. The reservoir is assumed to be heterogeneous, with a constant porosity $\phi$, and an isotropic log-normal permeability field $\mathbf{K}$.

The reservoir coarse mesh $\mathcal{M}_{r}$ is the uniform topologically Cartesian mesh of size $M_{x} \times M_{y} \times M_{z}$ with $M_{x}=$ $M_{y}=51$ and $M_{z}=3$. The nearwell subdomain $\Omega_{w}$ is the restriction of $\Omega_{r}$ to $(x, y) \in\left(-L_{w}, L_{w}\right) \times\left(-L_{w}, L_{w}\right)$ with $L_{w}=\frac{M_{w}}{2 \times 51} L$ for a given $M_{w} \in \mathbb{N}^{*}$. The nearwell mesh $\mathcal{M}_{w}$ is obtained by subdivision of all coarse cells in the subdomain $\Omega_{w}$ by a factor 3 in each direction $x, y, z$ leading to 27 fine cells by coarse cell. The isotropic log-normal permeability $\mathbf{K}$ is first defined on the uniform fine grid of size $3 M_{x} \times 3 M_{y} \times 3 M_{z}$ and upscaled in the coarse cells of $\mathcal{M}_{r}$ by simple averaging. The vertical well is perforated in the single bottom cell of centre $x=y=0$ at the coarse level and in the 3 refined cells of centres $x=y=0$ contained in this coarse cell at the fine level.

In both test cases, the relative permeabilities are defined by the following Corey's law

$$
k_{r}^{(\alpha)}\left(S^{(\alpha)}\right)=\left\{\begin{array}{rll}
0 & \text { if } \quad \bar{s}^{(\alpha)}<0, \\
1 & \text { if } \quad \bar{s}^{(\alpha)}>1, \\
\left(\bar{s}^{(\alpha)}\right)^{2} & \text { if } \quad 0 \leq \bar{s}^{(\alpha)} \leq 1,
\end{array} \quad \text { with } \bar{s}^{(\alpha)}=\frac{s^{(\alpha)}-s_{r}^{(\alpha)}}{1-s_{r}^{(1)}-s_{r}^{(2)}}, \alpha=1,2\right.
$$

using the residual saturations $s_{r}^{(\alpha)}, \alpha=1,2$.

\subsection{Gas injection test case}

The reservoir is the spherical cap defined by $L=2500 \mathrm{~m}, H=30 \mathrm{~m}, b=300 \mathrm{~m}$, with constant porosity $\phi=0.2$, and the permeability field $\mathbf{K}$ exhibited in figure 5 with values ranging from $1310^{-15}$ to $2310^{-11} \mathrm{~m}^{2}$. The nearwell region is obtained with $M_{w}=7$ corresponding to $7 \times 7 \times 3$ coarse cells.

We consider the previous Black Oil model with the two phases 1 (liquid) and 2 (gas) and the two components $1=\mathrm{H}_{2} \mathrm{O}$ and $2=\mathrm{CH}_{4}$. The reservoir is initially saturated by the liquid phase with $c_{2}^{(1)}=0$ and at the initial pressure $p_{\text {init }}^{(1)}=4010^{5} \mathrm{~Pa}$. Its boundary is assumed to be impervious. The gas phase is injected at the fixed bottom hole pressure $p_{\mathrm{bhp}}=6010^{5} \mathrm{~Pa}$ at $z_{\text {ref }}=0$ through the multi-perforated vertical well of radius $r_{w}=0.12$ $\mathrm{m}$. The thermodynamical equilibrium is given by Henry's law $f\left(p^{(2)}\right)=k_{H} p^{(2)}$ with $k_{H}=10^{-11} \mathrm{~Pa}^{-1}$. Both phases are assumed to be compressible with mass densities defined by

$$
\rho^{(2)}\left(p^{(2)}\right)=\frac{M}{R T} p^{(2)}
$$

for the gas phase, and by

$$
\rho^{(1)}\left(p^{(1)}\right)=\rho_{0}^{l}\left(1+c^{l} p^{(1)}\right),
$$

for the liquid phase, with the perfect gas constant $R=8.314 J . K^{-1} . \mathrm{mol}^{-1}$, the molar mass $M=0.016 \mathrm{Kg}$, the fixed temperature $T=323 \mathrm{~K}$, the liquid compressibility $c^{l}=410^{-10} \mathrm{~Pa}^{-1}$, and the liquid reference density $\rho_{0}^{l}=1000 \mathrm{Kg} \cdot \mathrm{m}^{-3}$. The liquid and gas viscosities are fixed to $\mu^{(1)}=510^{-3} \mathrm{~Pa} . \mathrm{s}$ and $\mu^{(2)}=10^{-4} \mathrm{~Pa} . \mathrm{s}$, and the relative permeabilities are given by Corey's law $(10)$ with $s_{r}^{(1)}=0.2$, and $s_{r}^{(2)}=0.1$. The capillary pressure is defined by Corey's law

$$
p_{c}\left(s^{(2)}\right)=p_{1}-p_{0} \log \left(\frac{1-s^{(2)}-s_{r}^{(1)}}{1-s_{r}^{(1)}}\right),
$$

with $p_{1}=510^{5} \mathrm{~Pa}$ and $p_{0}=110^{5} \mathrm{~Pa}$. As stated in the section 2 , the optimization of the parameters $\lambda_{\sigma}^{n}$ at each time step $n$ and for all $\sigma \in \mathcal{F}_{r w}$ defining the Robin nearwell interface conditions is based on the linear parabolic 
equation for the liquid pressure

$$
c^{l} \phi \partial_{t} p-\operatorname{div}\left(-\frac{\mathbf{K}}{\mu^{(1)}} \nabla p\right)=0 .
$$

In order to obtain an analytical formula for the parameters $\lambda_{\sigma}^{n}$, it is further simplified by integration on one coarse time step $\Delta t^{n}$, not taking into account the local time stepping. In addition, we will consider a $1 \mathrm{D}$ approximation set on the reservoir domain $(0, L)$ with the nearwell subdomain defined by $\left(L-L_{w}, L_{w}\right)$, using the constant porosity $\phi$ and freezing the value of the permeability at the interface denoted by $K_{\sigma}$. It leads to the following $1 \mathrm{D}$ elliptic equation

$$
p-\left(c_{\sigma}^{n}\right)^{2} \partial_{x x} p=0
$$

with $c_{\sigma}^{n}=\sqrt{\frac{K_{\sigma} \Delta t^{n}}{c^{l} \mu^{(1)} \phi}}$, and an homogeneous Neumann condition at $x=0$. The optimal value of the parameter $\lambda_{\sigma}^{n}$ can be computed in such a way that the DDM algorithm converges in two iterations for this simplified problem (see [8] for a detailed description of optimized Robin Schwarz methods). We obtain

$$
\lambda_{\sigma}=\frac{K_{\sigma}}{c_{\sigma}^{n}} \frac{a^{2}-1}{a^{2}+1}, \quad 1<a=\exp \left(\frac{L-L_{w}}{c_{\sigma}^{n}}\right) .
$$

It is expected that this simple analytical computation of the parameters $\lambda_{\sigma}^{n}$ will suffice to ensure a good convergence even when applied to our 3D heterogeneous reservoir, with local time stepping, and for our two phase flow problem.

Figure 6 exhibits the convergence of the optimized Robin-Neumann DDM iterations with local time stepping in the nearwell subdomain using the coarse time stepping $t^{0}=0, t^{1}=1, t^{2}=11, t^{3}=21, t^{4}=31, t^{5}=41$, $t^{6}=51, t^{7}=61$ days, and a local time stepping obtained by subdivision of each coarse time step into 10 subtime steps. Most of the time, the convergence is obtained in roughly 2 or at most 3 iterations for a stopping criteria defined by (8) with $\epsilon=10^{-2}$.

Next the solutions obtained with the following five algorithms are compared to the reference solution obtained using the LGR mesh with the global fine time stepping.

(i) The Robin-Neumann DDM algorithm with the stopping criteria (8) $\epsilon=10^{-2}$, using a $\theta$ scheme for the time integration in the coarse reservoir mesh with $\theta=1$ at the first coarse time step $\left(t^{0}, t^{1}\right)$ and with $\theta=\frac{3}{5}$ for the subsequent coarse time steps.

(ii) The Robin-Neumann DDM algorithm with the stopping criteria (8) $\epsilon=10^{-2}$, using the Euler implicit scheme $\theta=1$ on the coarse mesh for all coarse time steps.

(iii) The Robin-Neumann DDM algorithm with one DDM iteration and the Euler implicit scheme $\theta=1$ on the coarse mesh for all coarse time steps.

(iv) The windowing algorithm obtained by a sequential computation in time of the reservoir and nearwell solutions using a Dirichlet interface condition at the nearwell reservoir interface and the fixed bottom hole pressure $p_{r, b h p}=p_{b h p}$ at the well interface (D-windowing).

(v) The windowing algorithm obtained by a sequential computation in time of the reservoir and nearwell solutions using Neumann interface condition at the nearwell reservoir interface and the fixed bottom hole pressure $p_{r, b h p}=p_{b h p}$ at the well interface (N-windowing).

The well cumulative gas flow rate as a function of time obtained with the five above algorithms is exhibited in figure 7. Figure 8 exhibits the gas saturation solution obtained at final time for the reference solution and for the five algorithms, and figure 9 plots the gas saturation error at final time w.r.t. the reference solution. It clearly shows the large improvement provided by the Robin-Neumann DDM algorithms compared with the classical windowing approaches which are not able to reproduce accurately the solution. This improvement is already clear thanks to the Robin condition at the first DDM iteration for roughly speaking the same cost than the windowing algorithm. This solution is slightly improved with the subsequent iterations of algorithm (i) or (ii) using the stopping criteria (8) with $\epsilon=10^{-2}$. We also note the improvement provided by algorithm (i) compared with (ii) thanks to the more accurate time integration on the coarse reservoir mesh.

To evaluate the CPU times, let us denote by NLoc the number of cells in the nearwell subdomain, by NLGR the number of cells in the LGR grid, NCoarse the number of reservoir coarse cells, by Niter the number of DDM iterations, and by nlts the number of subtime steps. Then, the ratio of the CPU time obtained using the fine time step on the LGR grid by the CPU time obtained with the Robin-Neumann DDM algorithm can 
be estimated as follows

$$
C P U_{\text {ratio }}=\frac{\operatorname{nlts} \times(\text { NLGR })^{\beta}}{\text { Niter } \times\left(\text { nlts } \times(\text { NLoc })^{\beta}+(\text { NCoarse })^{\beta}\right)},
$$

assuming a complexity at each time step proportional to $N^{\beta}$ w.r.t. the number of cells $N$.

In the case of algorithm (iii) with nlts $=10$, NLGR $=11625$, NCoarse $=7803$, NLoc $=4065$, Niter $=1$, we obtain the estimation $C P U_{\text {ratio }}=2.4$ for $\beta=1, C P U_{\text {ratio }}=2.9$ for $\beta=1.2$, to be compared with our numerical experiment reported in the table below.

\begin{tabular}{|l|l|}
\hline Algorithms & CPU (s) \\
\hline LGR grid with global fine time step & 21.9 \\
\hline Robin-Neumann DDM with one iteration (iii) & 9.64 \\
\hline
\end{tabular}

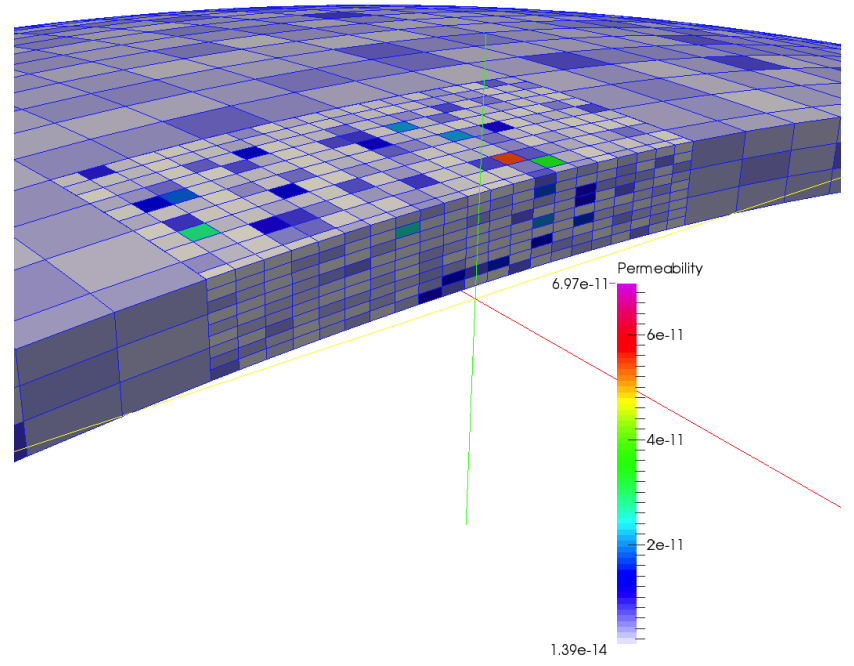

FiguRE 5. Log-normal permeability field upscaled on the coarse cells.

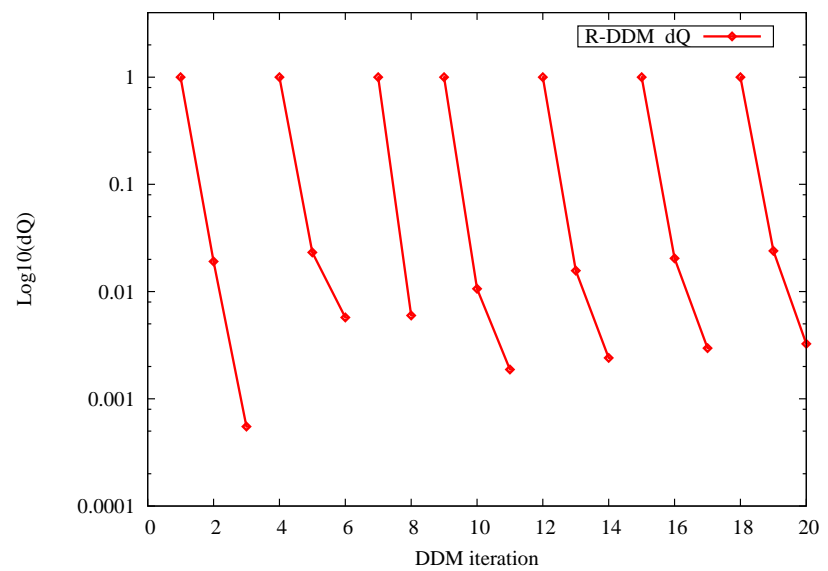

Figure 6. Convergence of $d Q$ (defined in (8)) for the Robin-Neumann DDM algorithm. 

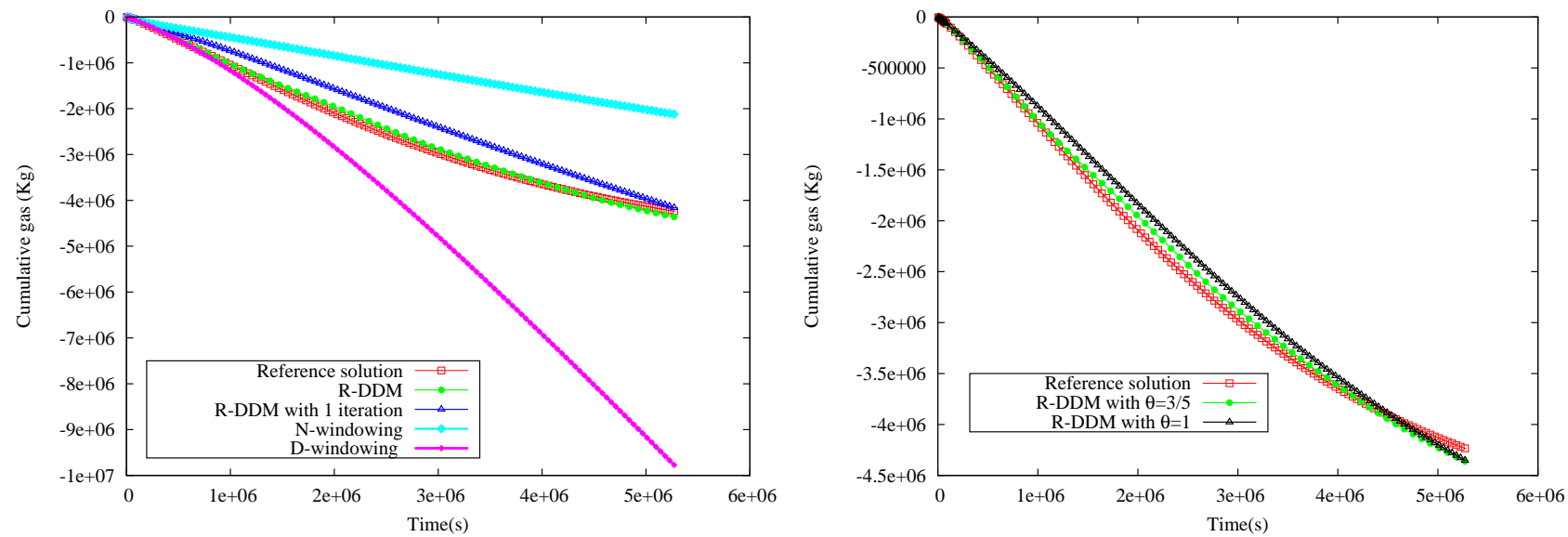

FiguRE 7. Left: comparison of the cumulative gas flow rate as a function of time $t \in(0,61$ days) obtained by the reference LGR algorithm with global fine time stepping (in red) and the algorithms (i) in green, (iii) in blue, (iv) in pink, and (v) in light blue. Right: Comparison of the cumulative gas flow rate as a function of time $t \in(0,61$ days $)$ obtained by the reference LGR algorithm with global fine time stepping in red and the algorithms (i) in green and (ii) in black. 

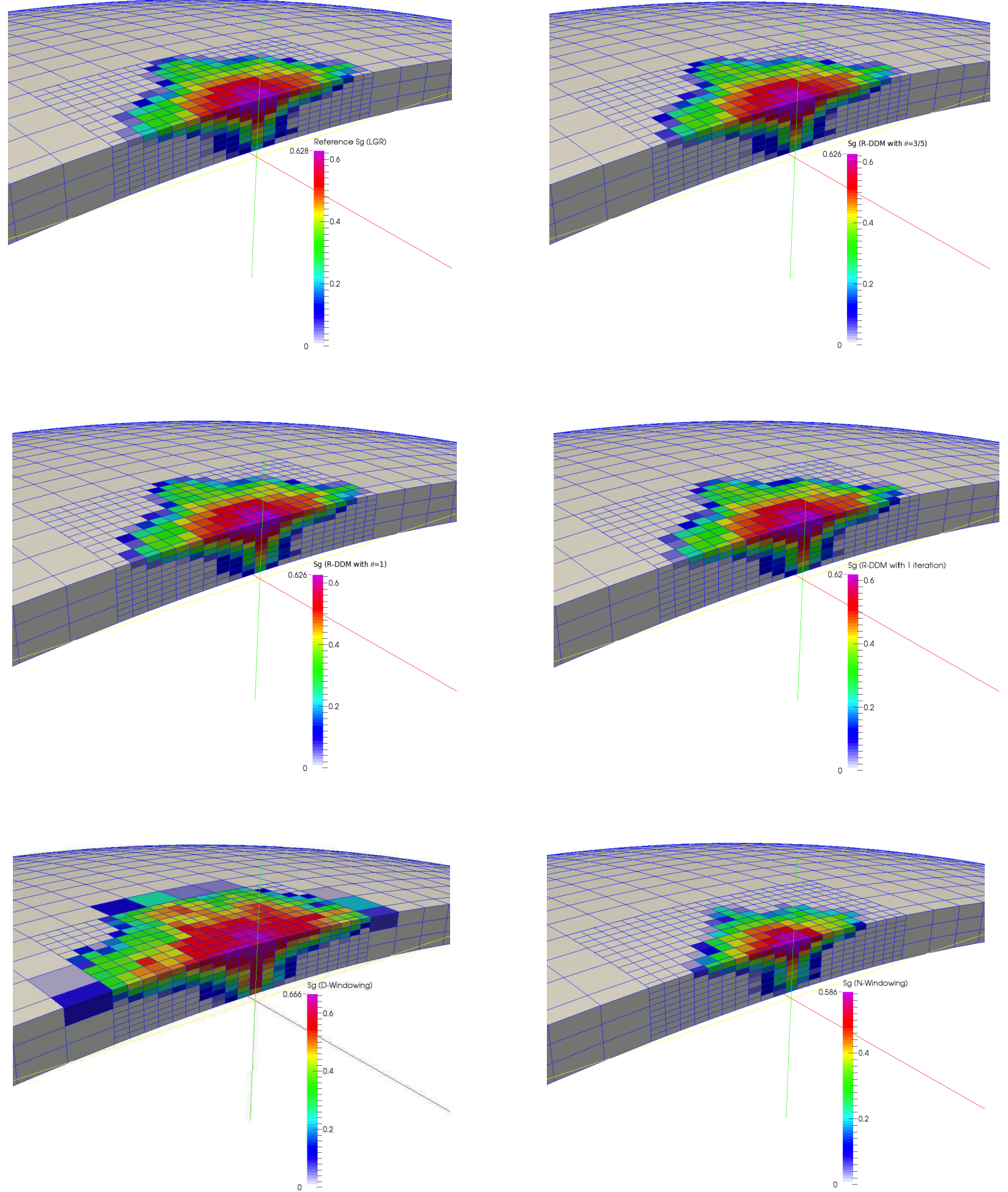

Figure 8. Gas saturation obtained at final time $t=61$ days, from top to bottom and from left to right, by: the reference LGR algorithm with global fine time stepping (LGR), and algorithms (i) (R-DDM with $\theta=3 / 5$ ), (ii) (R-DDM with $\theta=1$ ), (iii) (R-DDM with 1 iteration), (iv) (Dwindowing), and (v) (N-windowing) 

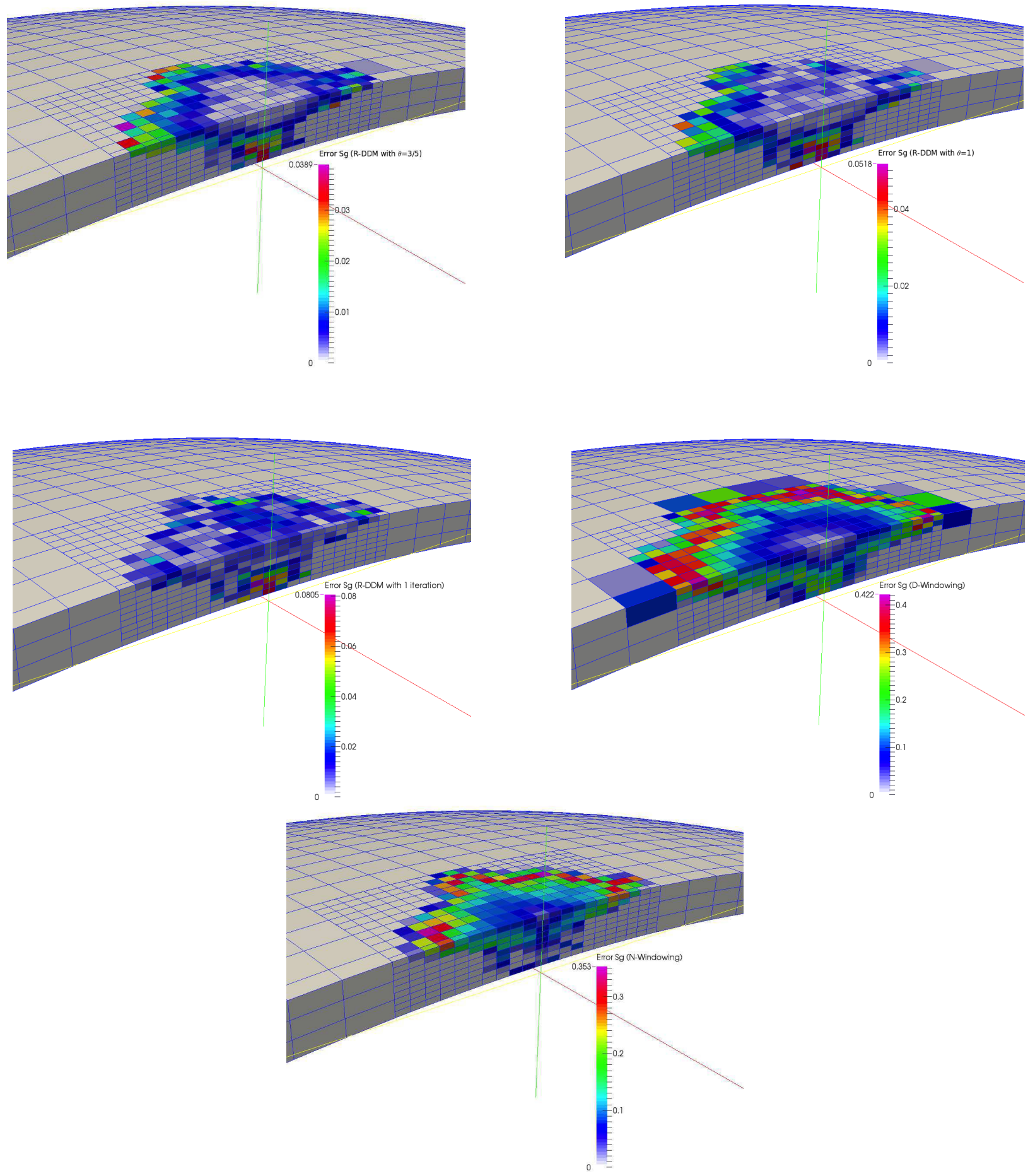

Figure 9. Gas saturation error compared with the reference solution obtained at final time $t=61$ days, from top to bottom and from left to right, by: (i) (R-DDM with $\theta=3 / 5$ ), (ii) (R-DDM with $\theta=1$ ), (iii) (R-DDM with 1 iteration), (iv) (D-windowing), and (v) (Nwindowing)

\subsection{Gas-Condensate model}

This second test case considers a gas-condensate model with phase 1 corresponding to the gas phase, phase 2 corresponding to the oil phase, and the components 1 and 2 corresponding respectively to light and heavy hydrocarbons (HC). The reservoir domain is the spherical cap defined by $L=250 \mathrm{~m}, H=30 \mathrm{~m}, b=30 \mathrm{~m}$, with constant porosity $\phi=0.2$, and the permeability field $\mathbf{K}$ exhibited in figure 10 with values ranging from 
$1.910^{-15}$ to $610^{-12} \mathrm{~m}^{2}$. The nearwell region is obtained with $M_{w}=3$ corresponding to $3 \times 3 \times 3$ coarse cells. It is initially saturated by the undersaturated gas phase at the initial pressure $p_{\text {init }}=3410^{5} \mathrm{~Pa}$ and with the initial heavy $\mathrm{HC}$ mass fraction $c_{2}^{(1)}=0.05$. The top and bottom boundaries are assumed to be impervious and the pressure is fixed to $3410^{5} \mathrm{~Pa}$ at the remaining boundaries with an input gas saturation equal to $s^{(1)}=1$ and an input mass fraction equal to $c_{2}^{(1)}=0.05$.

The vertical well of radius $r_{w}=0.12 \mathrm{~m}$ is a production well with imposed bottom hole pressure fixed to $p_{\mathrm{bhp}}=1010^{5} \mathrm{~Pa}$ at the reference coordinate $z_{\text {ref }}=0$. The thermodynamical equilibrium is defined by the following function giving the heavy $\mathrm{HC}$ mass fraction at equilibrium in the presence of both phases:

$$
f\left(p^{(2)}\right)= \begin{cases}\frac{p^{(2)}-p_{1}^{g}}{p_{3}^{g}-p_{1}^{g}} \bar{c}_{3}+\frac{p^{(2)}-p_{3}^{g}}{p_{1}^{g}-p_{3}^{g}} \bar{c}_{1} \text { if } \quad p^{(2)} \leq p_{3}^{g}, \\ \frac{p^{(2)}-p_{2}^{g}}{p_{3}^{g}-p_{2}^{g}} \bar{c}_{3}+\frac{p^{(2)}-p_{3}^{g}}{p_{2}^{g}-p_{3}^{g}} \bar{c}_{2} \text { if } \quad p^{(2)} \geq p_{3}^{g}\end{cases}
$$

where $p_{1}^{g}=1010^{5}, p_{2}^{g}=3410^{5}, p_{3}^{g}=2910^{5} \mathrm{~Pa}$, and $\bar{c}_{1}=10^{-2}, c_{2}=10^{-1}, \bar{c}_{3}=210^{-2}$.

The mass densities of both phases are defined by

$$
\rho^{(1)}\left(p^{(1)}\right)=\rho_{0}^{g}+c^{g} p^{(2)}
$$

for the gas phase, and by

$$
\rho^{(2)}\left(p^{(2)}\right)=\rho_{0}^{l}=800 \mathrm{Kg} \cdot \mathrm{m}^{-3},
$$

for the liquid phase, with the gas reference density $\rho_{0}^{g}=400 \mathrm{Kg} \cdot \mathrm{m}^{-3}$ and the gas compressibility $c^{g}=110^{-5}$ $\mathrm{Kg} \cdot \mathrm{m}^{-3} \mathrm{~Pa}^{-1}$. The liquid and gas viscosities are fixed to $\mu^{(2)}=110^{-3} \mathrm{~Pa}$.s and $\mu^{(1)}=10^{-4}$ Pa.s respectively, and their relative permeabities are given by Corey's law (10) with $s_{r}^{(1)}=0.2$ and $s_{r}^{(2)}=0.1$. The capillary pressure is assumed to be negligeable in this test case. The choice of the optimized Robin parameters $\lambda_{\sigma}^{n}$ is based, as in the previous test case, on a simple constant coefficient 1D elliptic equation on the gas pressure set on the domain $(0, L)$. Taking into account the modified Dirichlet boundary condition at $x=0$, we obtain

$$
\lambda_{\sigma}^{n}=\frac{K_{\sigma}}{c_{\sigma}^{n}} \frac{a^{2}-1}{a^{2}+1}
$$

with $1<a=\exp \left(\frac{L_{w}}{c^{n}}\right)$.

Figure 11 exhibits the convergence of the Robin-Neumann DDM iterations using the coarse time stepping $t^{0}=0, t^{1}=1, t^{2}=11, t^{3}=21, t^{4}=31, t^{5}=41, t^{6}=51, t^{7}=61, t^{8}=71, t^{9}=81, t^{10}=91, t^{11}=100$ days, and a local time stepping obtained by subdivision of each coarse time step into 10 subtime steps. The convergence is always obtained in two iterations for a stopping criteria defined by (8) with $\epsilon=10^{-2}$.

Next, the solutions obtained with the following four algorithms are compared to that obtained with the reference LGR algorithm with global fine time stepping.

(i) The Robin-Neumann DDM algorithm with the stopping criteria (8) $\epsilon=10^{-2}$,

(ii) The Robin-Neumann DDM algorithm with one iteration

(iii) The windowing algorithm obtained by a sequential computation in time of the reservoir and nearwell solutions using a Dirichlet interface condition at the nearwell reservoir interface and the fixed bottom hole pressure $p_{r, b h p}=p_{b h p}$ at the well interface (D-windowing).

(iv) The solution obtained on the LGR mesh $\mathcal{M}^{\text {lgr }}$ using the coarse time stepping (without LTS).

The well oil flow rate as a function of time obtained with the above four algorithms is exhibited in figure 12 . Figure 13 exhibits the oil saturation solution obtained at final time for the reference solution and for the four algorithms, and figure 14 plots the oil saturation error at final time w.r.t. the reference solution. Again, a large improvement is provided by the Robin-Neumann DDM algorithms compared with the windowing approaches. This improvement is already clear thanks to the Robin condition at the first DDM iteration but two iterations provides a much better solution. The gain in CPU time is reported in the following table compared with the reference LGR algorithm using the global fine time step. A factor of almost 3 is obtained with algorithm (i) which provides the best approximation.

\begin{tabular}{|l|l|}
\hline Algorithms & CPU (s) \\
\hline LGR grid with global fine time step & 18.11 \\
\hline Robin-Neumann DDM at convergence (i) & 6.95 \\
\hline
\end{tabular}




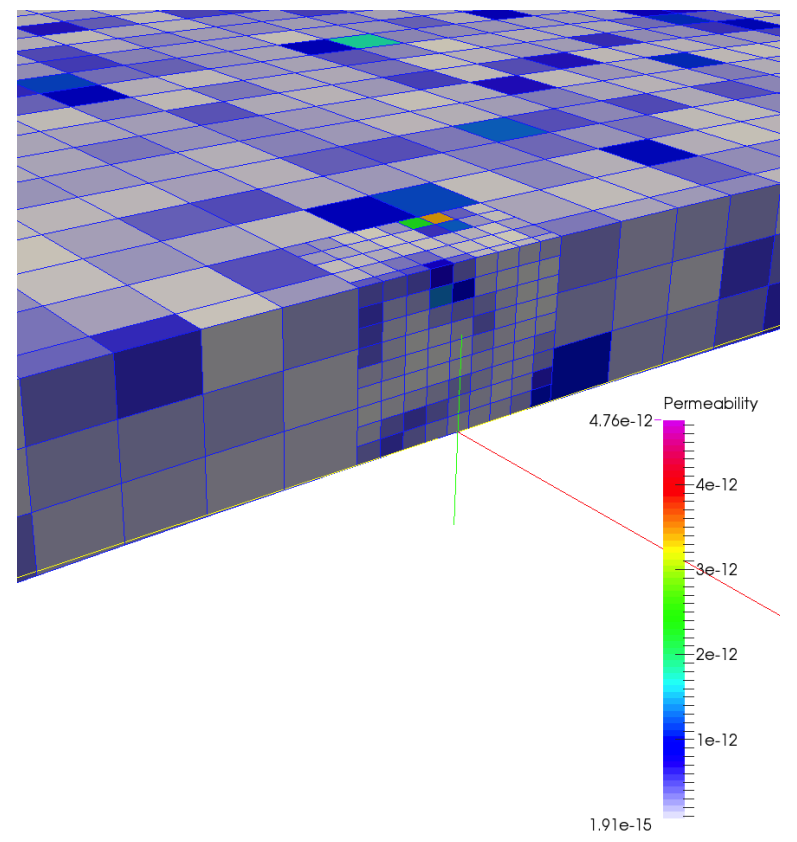

FiguRE 10. Log-normal permeability field upscaled in the coarse cells.

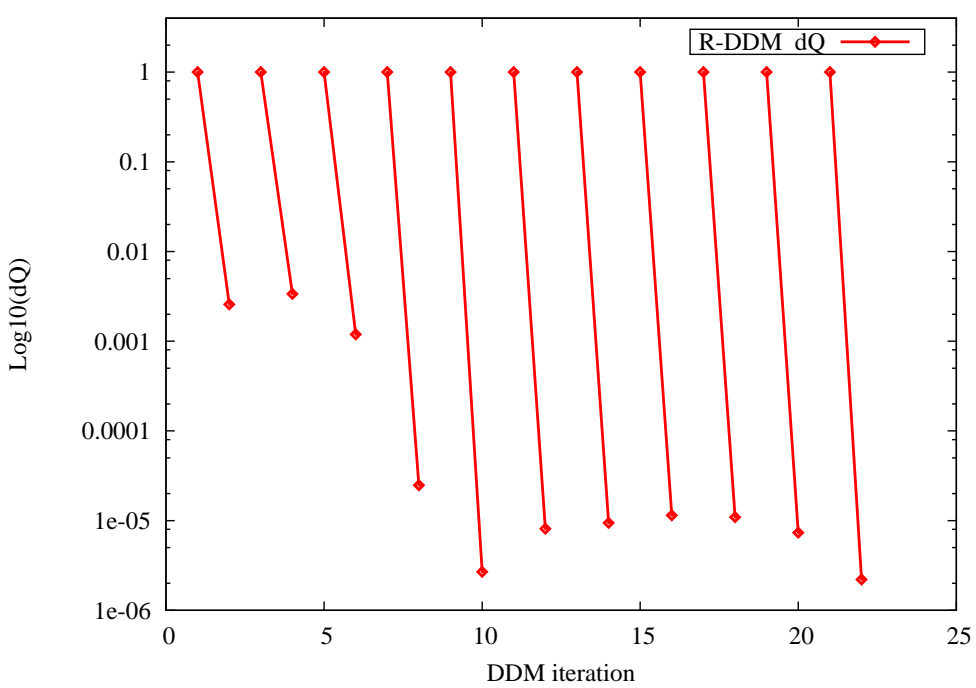

Figure 11. Convergence of $d Q$ (defined in (8)) for the Robin-Neumann DDM algorithm. 


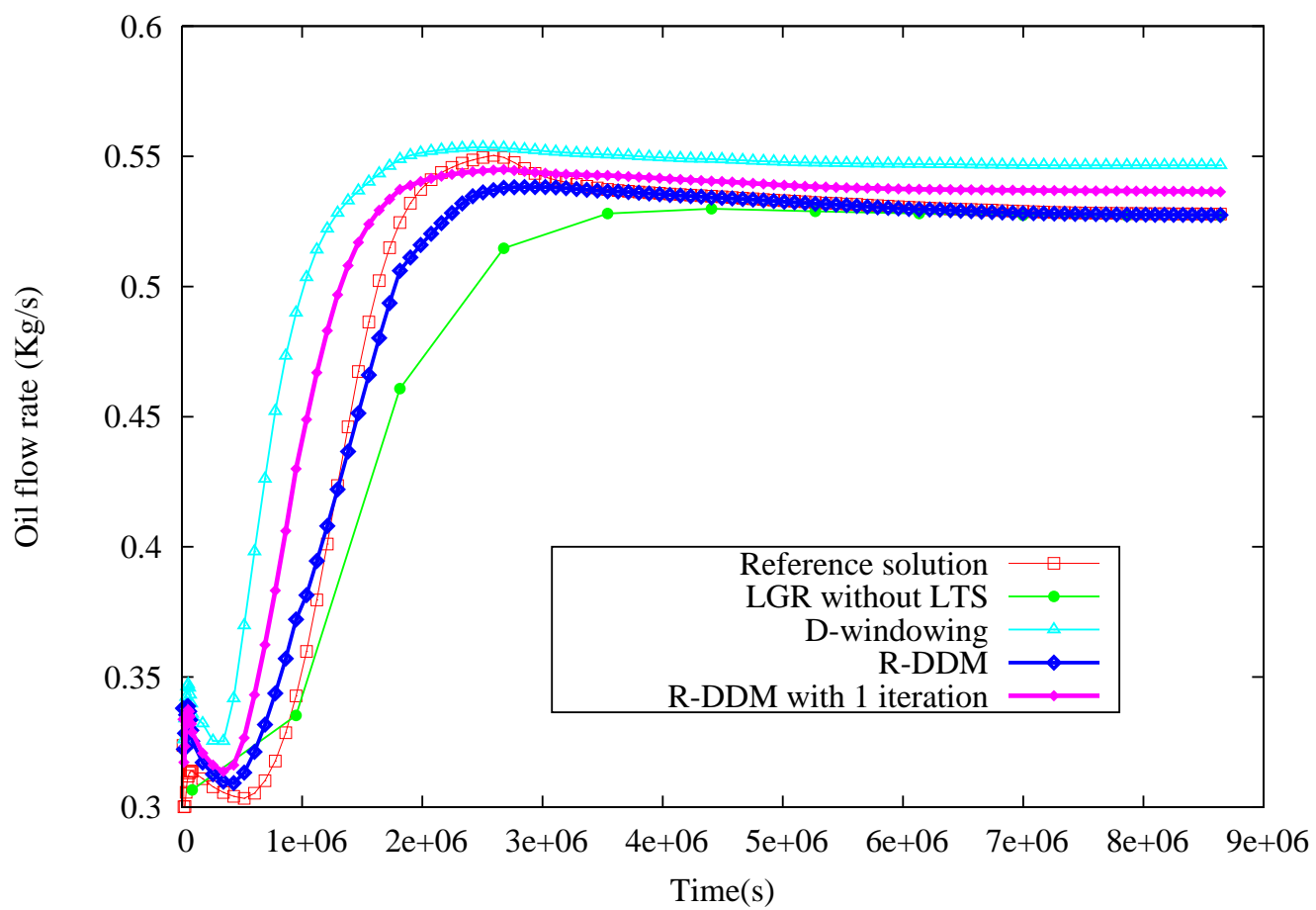

Figure 12. Comparison of the oil flow rate as a function of time $t \in(0,100$ days) obtained by the reference solution (in red) and the solutions of the four algorithms (i) (R-DDM) in blue, (ii) (R-DDM with 1 iteration) in pink, (iii) (D-windowing) in light blue, (iv) (LGR without LTS) in green. 

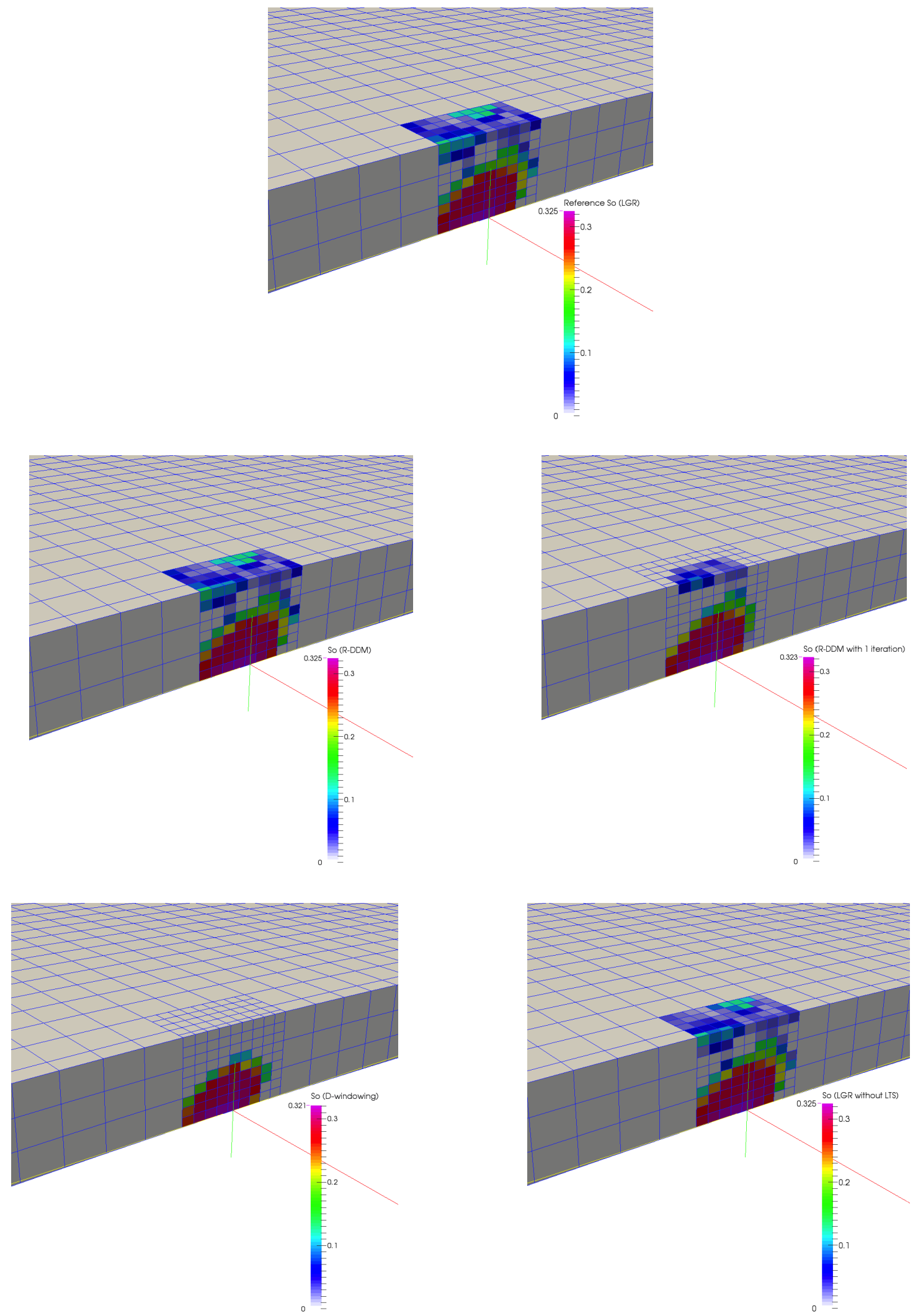

Figure 13. Oil saturation at final time $t=100$ days obtained from top to bottom and from left to right by: the reference solution (LGR), and algorithms (i) (R-DDM), (ii) (R-DDM with 1 iteration), (iii) (D-windowing), (iv) (LGR without LTS) 

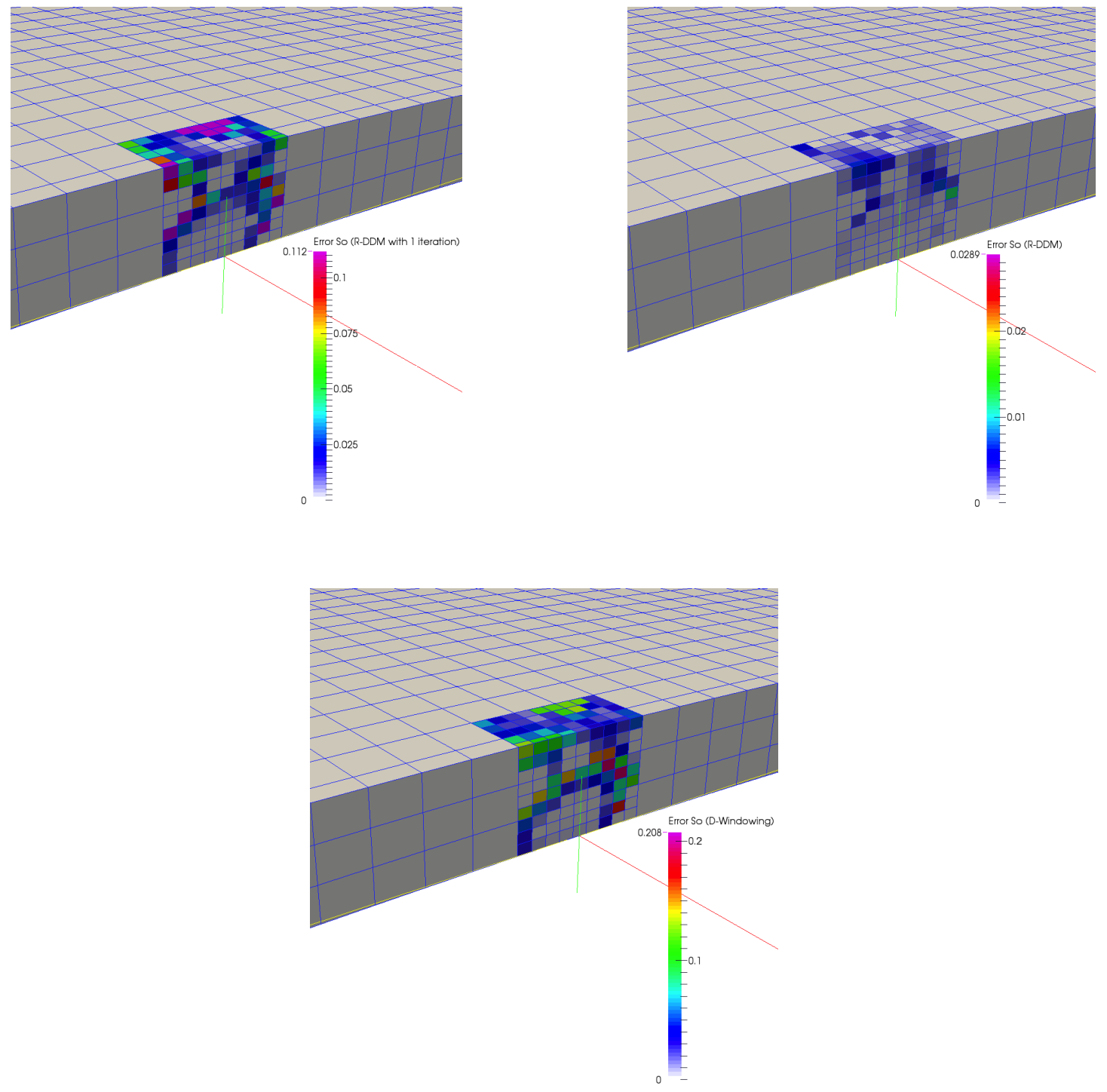

FIGURE 14. Oil saturation error compared with the reference solution obtained at time $t=100$ days from top to bottom and from left to right by the algorithms (ii) (R-DDM with 1 iteration), (i) (R-DDM), and (iii) (D-windowing).

\section{Conclusions}

A domain decomposition algorithm for a two phase Darcy flow model coupling nearwell regions locally refined in space and time with a coarser reservoir discretization has been presented. The algorithm is based on an optimized Schwarz method with a full overlap at the coarse level and using a decoupling of the pressure and saturation to obtain simple interface conditions. The main advantage of this approach is to apply to fully implicit discretizations of general multiphase flow models and to allow a simple optimization of the interface conditions based on a single phase flow equation.

The results obtained on 3D gas injection and gas-condensate models with a vertical multi-perforated injection or production well exhibit the good behaviour of the Robin-Neumann DDM algorithm which converges in roughly 2 iterations and provides a good accuracy compared with the LGR reference solution using the fine time stepping. The comparison with classical windowing approaches clearly shows a large improvement in terms of accuracy for a roughly double cost.

Compared with a global time stepping on the LGR grid, we observe important gains in CPU time provided that the number of d.o.f. in the locally refined zones is significantly smaller than the total number of d.o.f. 


\section{REFERENCES}

[1] ECLIPSE Software, Technical Description, Schlumberger, subsection local time stepping, section 29, page 579. n.d.

[2] I. Aavatsmark. An introduction to multipoint flux approximations for quadrilateral grids. Computational Geosciences, 6:405$432,2002$.

[3] K. Aziz and A. Settari. Petroleum Reservoir Simulation. Applied Science Publishers, London, U.K, 1979.

[4] K. H. Coats. Implicit compositional simulation of single-porosity and dual-porosity reservoirs. Houston, Texas, 1989. SPE Symposium on Reservoir Simulation.

[5] R. Ewing, R. Lazarov, and P. Vassilevski. Finite difference schemes on grids with local refinement in time and space for parabolic problems i. derivation, stability, and error analysis. Computing, 45:193-215, 1990.

[6] R. Eymard, T. Gallout, and R. Herbin. Finite volume methods. In P. Ciarlet and J. Lions, editors, Solution of Equation in $\mathbb{R}^{n}$ (Part 3), Techniques of Scientific Computing (Part 3), volume 7 of Handbook of Numerical Analysis, pages 713 - 1018. Elsevier, 2000.

[7] I. Faille, F. Nataf, F. Willien, and S. Wolf. Two local time stepping schemes for parabolic problems. ESAIM proceedings, 29:58-72, 2009.

[8] M. J. Gander. Optimized schwarz methods. SIAM J. Numer. Anal., 44:699-731, 2006.

[9] M. Mlacnik. Using Well Windows in Full Field Reservoir Simulations. PhD thesis, University of Leoben, 2002.

[10] M. Mlacnik and Z. Heinemann. Using well windows in full-field reservoir simulation. volume 6, pages $275-285$. SPE Reservoir Evaluation and Engineering, 2003.

[11] D. Peaceman. Fundamentals of Numerical Reservoir Simulations. Elsevier, 1977.

[12] J. A. Trangenstein and J. B. Bell. Mathematical structure of the black-oil model for petroleum reservoir simulation. SIAM Journal on Applied Mathematics, 49:749-783, 1989. 